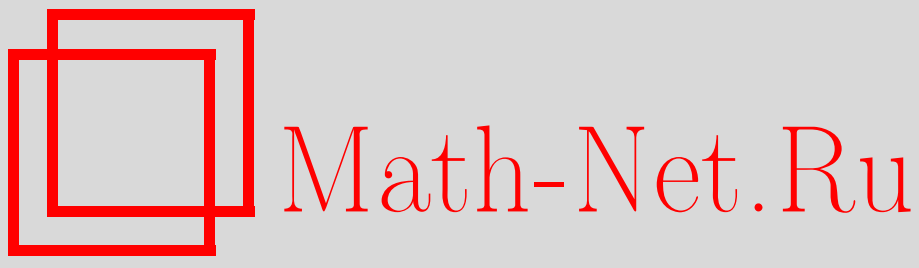

И. И. Аргатов, С. А. Назаров, Асимптотическое решение задачи Синьорини с препятствием на тонком продолговатом множестве, Матем. сб., 1996, том 187, номер 10, 3-32

DOI: https://doi.org/10.4213/sm162

Использование Общероссийского математического портала Math-Net.Ru подразумевает, что вы прочитали и согласны с пользовательским соглашением

http://www . mathnet.ru/rus/agreement

Параметры загрузки:

IP: 35.173 .137 .237

26 апреля 2023 г., 02:29:59 
УДК 517.953

\author{
И.И. Аргатов, С.А. Назаров
}

\title{
Асимптотическое решение задачи Синьорини с препятствием на тонком продолговатом множестве
}

\begin{abstract}
Изучается задача Синьорини для уравнения Пуассона при условии, что односторонние связи накладьваются на узкой (шириной $O(\varepsilon)$ ) кольцевой полоске $\Gamma_{\varepsilon}$ границы. Асимптотический анализ позволяет получить результирующее вариационное неравенство на контуре $\Gamma$, к которому стягивается $\Gamma_{\varepsilon}$ при $\varepsilon \rightarrow 0$. Отыскиваются приближенные решения (различной точности) результирующего неравенства и при их участии строится и обосновьвается асимптотика решения исходной задачи Синьорини.

Библиографиия: 34 названия.
\end{abstract}

\section{§1. Постановка задачи и предварительное описание результатов}

1.1. Область и вариационное неравенство. Пусть $\Omega$ - область в $\mathbb{R}^{3}$, ограниченная гладкой (класса $C^{\infty}$ ) замкнутой поверхностью $\partial \Omega$, а $\Gamma$-простой гладкий замкнутый контур на $\partial \Omega$ длиной $l$. Для упрощения формул будем считать, что $\Gamma-$ экватор (сечение $\partial \Omega$ плоскостью $x_{3}=0$ ) и что в его окрестности $\mathscr{U} \subset \mathbb{R}^{3}$ граница $\partial \Omega$ совпадает со связной цилиндрической поверхностью $\Gamma \times \mathbb{R}$. Сузив при необходимости множество $\mathscr{U}$, зададим в нем естественные криволинейные координаты $(s, z, n)$, где $z=x_{3}, n$ - расстояние до $\partial \Omega$ вдоль внутренней нормали, а под $s$ подразумевается длина дуги на $\Gamma$ (т.е. $s \in[0, l)$ или, несколько вольно, $s \in \Gamma$ ). Введем в рассмотрение узкую экваториальную полоску

$$
\Gamma_{\varepsilon}=\left\{x \in \partial \Omega: s \in \Gamma,-\varepsilon h_{-}(s)<z<\varepsilon h_{+}(s)\right\},
$$

где $\varepsilon$ - малый положительный параметр, $h_{ \pm} \in C^{\infty}(\Gamma)$, причем $h=h_{+}+h_{-}$ - положительная функция. Наконец, $\Sigma$ - открытое подмножество $\partial \Omega$ такое, что $\operatorname{mes}_{2} \Sigma>0$ и $\bar{\Sigma} \cap \bar{\Gamma}_{\varepsilon}=\varnothing$ при $\varepsilon \in\left(0, \varepsilon_{\Sigma}\right), \varepsilon_{\Sigma}>0$ (рис. 1$)$.

Задача Синьорини состоит в нахождении функции $u$, удовлетворяюшей следуюшим соотношениям:

$$
\begin{gathered}
-\Delta_{x} u(\varepsilon, x)=f(\varepsilon, x), \quad x \in \Omega ; \\
u(\varepsilon, x)=0, \quad x \in \Sigma ; \\
\partial_{n} u(\varepsilon, x)=0, \quad x \in \partial \Omega \backslash\left(\bar{\Sigma} \cup \bar{\Gamma}_{\varepsilon}\right) ; \\
u(\varepsilon, x) \geqslant \varphi(\varepsilon, s), \quad \partial_{n} u(\varepsilon, x) \leqslant 0, \\
(u(\varepsilon, x)-\varphi(\varepsilon, s)) \partial_{n} u(\varepsilon, x)=0, \quad x \in \Gamma_{\varepsilon} ;
\end{gathered}
$$

здесь $\partial_{n}=\partial / \partial n$, а $f$ и $\varphi$ - заданные функции, к которым по мере необходимости будут предъявляться дополнительные требования.

Фиксируем некоторое $\varepsilon$ и допустим, что $f(\varepsilon, \cdot) \in L_{2}(\Omega), \varphi(\varepsilon, \cdot) \in L_{2}(\Gamma)$. Известно (см. [1]-[3] и др.), что задача (1.2)-(1.5) имеет единственное решение 
$u(\varepsilon, \cdot) \in W_{2}^{1}(\Omega)$ и может быть переформулирована как задача о минимуме функционала

$$
\left(\nabla_{x} u, \nabla_{x} u\right)_{\Omega}-2(f, u)_{\Omega}
$$

на выпуклом замкнутом множестве

$$
K=\left\{u \in W_{2}^{1}(\Omega): u=0 \text { п.в. на } \Sigma, u \geqslant \varphi \text { п.в. на } \Gamma_{\varepsilon}\right\}
$$

или как вариационное неравенство

$$
\left(\nabla_{x} u, \nabla_{x} u-\nabla_{x} \zeta\right)_{\Omega} \leqslant(f, u-\zeta)_{\Omega} \quad \forall \zeta \in K .
$$

Здесь $\nabla_{x}=\operatorname{grad},(\cdot, \cdot)_{\Omega}-$ скалярное произведение в $L_{2}(\Omega)$ (векторные и скалярные пространства не различаются в обозначениях).

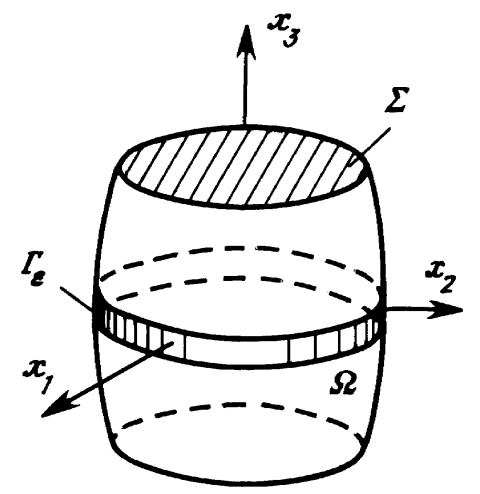

Рис. 1

1.2. Краткая история проблемы. Изучение асимптотических решений вариационных неравенств с различньми сингулярными возмущениями проводилось в [4]-[10] и др. Именно, при помощи асимптотического анализа (в рамках методов срашиваемых или составных разложений) исходная задача с мальм параметром $\varepsilon$ разбивалась на несколько последовательно решаемых, более простых задач, не содержащих $\varepsilon$ и называемых предельными. В случае задания односторонних связей (условий типа (1.5)) на малых множествах для части предельных задач отыскивались явные решения, зависяшие от ряда свободных констант, а для всего набора $N$ свободных констант выводилось результирующее вариационное неравенство (алгебраическое: оно ставится на выпуклом конусе в $\mathbb{R}^{N}$ ). После нахождения констант из результируюшего неравенства весь произвол в выборе решений предельных задач устранялся и их удавалось “склеить" в глобальное асимптотическое приближение $U(\varepsilon, \cdot)$ к решению $u(\varepsilon, x)$ исходной сингулярно возмушенной задачи, а разность $u(\varepsilon, x)-U(\varepsilon, x)$ характеризовалась на основе какой-либо априорной оценки. В целом эта схема сохраняется для задачи (1.2)-(1.5), но имеются и существенные отличия: результирующее неравенство в качестве неизвестной содержит функцию на $\Gamma$, причем в нем фигурирует интегральньй оператор на $Г$ и остается (большой) параметр $|\ln \varepsilon|$, а значит, оно само нуждается в асимптотической обработке.

Подобные эффекты проявляются и в линейных задачах при сингулярных возмушениях, сконцентрированных на тонких продолговатых множествах. Простейшим, но типичным, примером служит задача Дирихле для оператора Лапласа в 
трехмерной области с узкой полостью (см. [11]-[14] и др., а также [15], где границе полости дозволено меняться периодически, а периоду - сравняться по порядку с малым диаметром $O(\varepsilon)$ сечения). В таких задачах в качестве результирующего возникает интегральное уравнение на "оси" Г полости. В него входит параметр $|\ln \varepsilon|$ и в [11]-[14] предложено несколько методик построения асимптотических решений, приводяших к разложениям по обратньм степеням $|\ln \varepsilon|$ или к более точньм разложениям со “степенными” остатками $O\left(\varepsilon^{p}\right)$. К сожалению, нелинейность задачи (1.2)-(1.5) отвергает большинство подходов и оставляет единственно возможной “логарифмическую асимптотику”.

Основное отличие асимптотических построений в задачах с односторонними связями состоит в том, что вместе с асимптотикой решений требуется найти и множества, на которых реализуется то или иное краевое условие. Обычно удается оправдать только асимптотическое представление решения, а информация о структуре зоны контакта остается неизвестной - множества, на которых можно с уверенностью фиксировать тип краевого условия, не покрывают свободную поверхность (полоску $\Gamma_{\varepsilon}$ в случае задачи (1.2)-(1.5)). Настоящая работа не является исключением и, говоря о вариации зоны контакта, мы подразумеваем лишш уточнение заведомо неполных сведений о том, где на $\Gamma_{\varepsilon}$ осушествляется равенство $u=\varphi$, а где - равенство $\partial_{n} u=0$.

Напомним также работы [16]-[18], где задачи механики двумерных трещин интерпретировались как сингулярно возмушенные, выводились вариационные неравенства, похожие на те, с которыми мы столкнулись в данной работе, и на их основе формулировались критерии разрушения.

1.3. Структура статьи. В $\S 2$ мы строим формальную асимптотику решения задачи (1.2)-(1.5). Применяется метод срашиваемых асимптотических разложений (см. [19]-[21], [22, §6.5] и др.) и находятся главные члены внешнего и внутреннего разложений. Задача, описывающая плоский пограничный слой вблизи $\Gamma_{\varepsilon}$ (внутреннее разложение), решается явно. В качестве решений первой предельной задачи в $\Omega$ (внешнее разложение) берутся функции, обладаюшие логарифмическим ростом около $\Gamma$, т.е. вдоль контура $Г$ с некоторой плотностью $\gamma$ распределяется $\delta$-функция Дирака. Именно плотность $\gamma$ и является неизвестной в результируюшем вариационном неравенстве, возникаюшем как условие сращивания внешнего и внутреннего разложений.

В $\S 3$ мы сначала предлагаем простое асимптотическое решение $\gamma_{*}$ результирующего неравенства, а затем по нему конструируем глобальное асимптотическое приближение $U(\varepsilon, x)$ к истинному решению $u(\varepsilon, x)$ задачи (1.2)-(1.5) и занимаемся оценкой разности $R=u-U$. При этом теорема, обосновываюшая асимптотику $u \sim U$, формулируется так, что в центральной оценке остатка $R$ фигурирует норма невязки $\gamma_{*}$ в результируюшем неравенстве - порядок такой нормы (относительно $\varepsilon$ ) и определяет качество асимптотического приближения. Это обстоятельство позволяет не повторять обоснование асимптотики после ее уточнения в $\S 4$, где определяется второй член асимптотики плотности и одновременно находится вариация зоны контакта.

В $\S 5$ установленные оценки остатков в асимптотических формулах для решений переформулируются как теоремы о сходимости $u(\varepsilon, \cdot)$ при $\varepsilon \rightarrow+0$. В частности, данные задачи (1.2)-(1.5) удается подобрать так, чтобы пределом в $L_{2}(\Omega)$ функции $x \mapsto u(\varepsilon, x)$ оказалось (иррегулярное) решение смешанной краевой задачи в $\Omega$ с $\delta$-функцией, распределенной по $Г$. Наконец, в $\S 6$ кратко обсуждается 
случай $\Sigma=\varnothing$. Поясняется, как модифицировать нахождение асимптотического решения задачи (1.2)-(1.5) при отсутствии условий Дирихле (1.3). Оказьвается, что нарушение однозначной разрешимости первой предельной задачи (при $\Sigma=\varnothing$ и $\varepsilon=0$ на $\partial \Omega \backslash \Gamma$ остаются только условия Неймана) приводит к изменению результирующей задачи.

\section{§ 2. Главные члены внешнего и внутреннего разложений. Результирующее вариационное неравенство на $Г$}

2.1. Предварительное обсуждение задачи. Начнем с наиболее простого случая: функции $f$ и $\varphi$ в (1.2) и (1.5) не зависят от $\varepsilon$. В пределе при $\varepsilon \rightarrow+0$ множество (1.1) стягивается к контуру $\Gamma$, соотношения (1.5) исчезают, а уравнения (1.2)-(1.4) формируют первую предельную задачу

$$
\begin{gathered}
-\Delta_{x} v(x)=f(x), \quad x \in \Omega \\
v(x)=0, \quad x \in \Sigma ; \quad \partial_{n} v(x)=0, \quad x \in \partial \Omega \backslash(\bar{\Sigma} \cup \Gamma) .
\end{gathered}
$$

Пусть $f \in L_{p}(\Omega)$, где $p>3$. Тогда сушествует ограниченное решение $V$ задачи $(2.1)$, которое принадлежит $W_{p}^{2}(\mathscr{U} \cap \Omega)$ (отметим, что на линии $\partial \Sigma$ смены типа краевого условия grad $V$ имеет особенности, но все дальнейшие рассуждения относятся лишь к окрестности контура $Г$ ). Кроме того, имеет место вложение $W_{p}^{2}$ в $C^{1}$ и потому определен след $\psi$ функции $V$ на контуре $\Gamma, \psi \in C^{1}(\Gamma)$. Предположим, что $\varphi \in C^{1}(\Gamma)$ и обсудим несколько возможных ситуаций.

$1^{\circ}$. Если

$$
\psi(s)>\varphi(s) \quad \forall s \in \Gamma,
$$

то найдется такое $\varepsilon_{0}>0$, что при $\varepsilon \in\left(0, \varepsilon_{0}\right)$ на $\Gamma_{\varepsilon}$ оказывается верньм неравенство $V \geqslant \varphi$ (т.е. первое ограничение в (1.5)). При этом благодаря условию Неймана в (2.1) соблюдаются и остальные два требования из (1.5). Следовательно, в случае $\varepsilon \in\left(0, \varepsilon_{0}\right)$ решением задачи Синьорини (1.2)-(1.5) служит решение $V$ линейной задачи (2.1).

$2^{\circ}$. Пусть справедливо противоположное (2.2) неравенство

$$
\psi(s)<\varphi(s) \quad \forall s \in \Gamma
$$

Тогда при малом $\varepsilon$ первое условие в (1.5) не может выполняться для $V$. Далее будет пояснено (см. замечание 3.6 ), что в случае (2.3) решение задачи Синьорини при $\varepsilon \in\left(0, \varepsilon_{0}\right)$ совпадает с решением линейной смешанной краевой задачи, образованной добавлением к уравнениям (1.2)-(1.4) условий Дирихле

$$
u(\varepsilon, x)=\varphi(s), \quad x \in \Gamma_{\varepsilon} .
$$

Процедура построения асимптотики решений задач с данными Дирихле на тонких продолговатых множествах разработана в [11]-[14] (см. также [23, гл. 4] и $[24$, гл. 12, 13]).

$3^{\circ}$. В случае общего положения (ни одно из неравенств (2.2) или (2.3) не выполнено всюду на Г) асимптотический анализ включает в себя поиск зон контакта (на них в первом условии из (1.5) реализуется знак равенства). При этом возникают разнообразные частные задачи, описывающие возникновение или исчезновение 
зон контакта, их малые вариации и пр. (здесь пригодна классификация, предложенная в [8] для двумерных задач; см. также [5], [6]). Мы ограничимся рассмотрением следующей ситуации: множества

$$
\Xi^{ \pm}=\{s \in \Gamma: \pm \psi(s)< \pm \varphi(s)\}
$$

суть конечные наборы открытых дуг $\Xi_{n}^{ \pm}, n=1, \ldots, N$; дуги, помеченные одинаковыми символами “+" или “-”, не имеют общих концов; множество $\Xi^{0}=$ $\Gamma \backslash\left(\Xi^{+} \cup \Xi^{-}\right)$состоит из $2 N$ точек. Иными словами, разность $\psi-\varphi$ обращается в нуль лишь в изолированных точках, причем при переходе через эти точки обязательно меняет знак. Как будет показано далее, при таких ограничениях зона контакта $\Xi^{+}(\varepsilon)$ оказывается в некотором смысле мальм возмушением дуг $\Xi_{1}^{+}, \ldots, \Xi_{N}^{+}$.

2.2. Главный член внешнего разложения (дальнее поле). Применим метод сращиваемых асимптотических разложений (см. [19]-[22] и др.) и будем искать различные представления для решения $u(\varepsilon, x)$ на удалении от полоски $\Gamma_{\varepsilon}$ и в непосредственной близости к ней. Ясно, что главный член разложения решения $u$, подчиненного (1.2)-(1.4), должен удовлетворять (2.1). Поступая в соответствии c [11]-[14], позволим решению $v$ задачи (2.1) иметь логарифмические особенности на экваторе $\Gamma$, т.е. предположим, что

$$
v(x)=-\pi^{-1} \gamma(s) \ln r+O(1), \quad r \rightarrow 0 .
$$

Здесь $r=\left(z^{2}+n^{2}\right)^{1 / 2}$ - расстояние до $\Gamma$ в $\mathscr{U} \subset \mathbb{R}^{3}$, а $\gamma$ - функция, подлежашая дальнейшему определению. Утверждение об однозначной разрешимости задачи (2.1), (2.6) с заданньми $f$ и $\gamma$ можно вывести из общих результатов [22], [25], при этом $\gamma$ предписываются некоторые дифференциальные свойства. Однако для наших целей удобно интерпретировать сингулярное решение $v$ как решение задачи (2.1), в которой вдоль контура $Г$ распределена $\delta$-функция Дирака с плотностью $\gamma$, и ограничиться явньм интегральным представлением для $v$.

Обозначим через $G(\cdot ; s)$ обобшенную функцию Грина с особенностью в точке $\mathbf{s} \in \Gamma$ с координатой $s$ (в настояшем разделе приходится различать символы $\mathbf{s}$ и $s$ ). Эта функция удовлетворяет соотношениям

$$
\begin{gathered}
-\Delta_{x} G(x ; s)=0, \quad x \in \Omega \\
G(x ; s)=0, \quad x \in \Sigma ; \quad \partial_{n} G(x ; s)=0, \quad x \in \partial \Omega \backslash(\bar{\Sigma} \cup \mathbf{s}) \\
G(x ; s)=(2 \pi|x-\mathbf{s}|)^{-1}+g(x ; s), \quad\left|\nabla_{x}^{k} g(x ; s)\right| \leqslant c_{k, \delta}|x-\mathbf{s}|^{\delta-1-k} .
\end{gathered}
$$

$\mathrm{B}(2.7)|x-\mathbf{s}|$ - расстояние между точками $x \in \bar{\Omega}$ и $\mathbf{s} \in \Gamma$ в $\mathbb{R}^{3}, \nabla_{x}^{k} \zeta$-совокупность всех производных порядка $k$ функции $\zeta, k=0,1, \ldots$ и $\delta \in(0,1)$. Искомое решение $v$ есть сумма

$$
v(x)=V(x)+\int_{\Gamma} \gamma(t) G(x ; t) d t,
$$

где $V$ - ограниченное решение задачи (2.1).

2.3. Асимптотика сингулярного решения. Логарифмическая асимптотика (2.6) сосредоточенного на Г потенциала (интеграл из (2.8)) известна. При помощи регуляризации интеграла можно выписать и уточненную асимптотику. В частности, если $\gamma \in W_{\infty}^{1}(\Gamma)$, то в силу, например, [11]-[15] или [17]

$$
v(x)=-\pi^{-1} \gamma(s) \ln r+J \gamma(s)+\psi(s)+O(r|\ln r|), \quad r \rightarrow 0 .
$$


Пусть $\mathscr{G}$ - какая-нибудь первообразная функции $\Gamma \ni t \mapsto G(\mathbf{t}, s)$. Так как

$$
G(\mathbf{t}, s)=(2 \pi|\mathbf{t}-\mathbf{s}|)^{-1}+o\left(|\mathbf{t}-\mathbf{s}|^{\delta-1}\right), \quad \mathbf{t} \rightarrow \mathbf{s},
$$

то при $\mp t>\mp s$

$$
\mathscr{G}(t, s)= \pm(2 \pi)^{-1} \ln |t-s| \pm \mathscr{G}^{ \pm}(t, s) .
$$

Явное представление для фигурирующего в (2.9) интегрального оператора (см. [14], [15], [24]) имеет вид:

$$
\begin{aligned}
J \gamma(s)=(2 \pi)^{-1} \int_{\Gamma}(\gamma(t)-\gamma(s))|\mathbf{t}-\mathbf{s}|^{-1} d t+\int_{\Gamma} \gamma(t) g(s, t) d t \\
\quad+\pi^{-1} \ln 2-\mathscr{G}^{+}(s+0, s)-\mathscr{G}^{-}(s-0, s) .
\end{aligned}
$$

Далее, при обосновании асимптотики, понадобятся оценки остатка

$$
\widetilde{v}(x)=v(x)+\pi^{-1} \gamma(s) \ln r-J \gamma(s)-\psi(s)
$$

в представлении (2.9). Мы приведем два утверждения, различающиеся требованиями, предъявляемыми к плотности $\gamma$. Первое, более простое, будет применяться преимушественно; его доказательство, по сушеству, содержится в [11]-[15], требуется лишь незначительная модификация, связанная с переходом от внутреннего контура $\Gamma \subset \Omega$ к кривой $\Gamma$ на $\partial \Omega$. Второе утверждение получается конкретизацией общих результатов [22, гл. 11], [25, гл. 9], оно используется лишш однажды, в $\S 4$ (впрочем, в этой ситуации можно было бы повторить выкладки из [11]-[15], но мы предпочитаем избавить статью от громоздких вычислений, не относящихся к сути дела).

ПРЕДЛОЖЕНИЕ 2.1. Пусть $f \in L_{p}(\Omega)$ с некоторым $p>3$.

1) Если $\gamma \in W_{\infty}^{1}(\Gamma)$, то при $x \in \Omega \cap \mathscr{U}$ верна оценка

$$
r^{-1}|\widetilde{v}(x)|+\left|\nabla_{x} \widetilde{v}(x)\right| \leqslant c_{p}(1+|\ln r|)\left(\left\|f ; L_{p}(\Omega)\right\|+\left\|\gamma ; W_{\infty}^{1}(\Gamma)\right\|\right) .
$$

2) Если $\gamma \in W_{2}^{1}(\Gamma)$, то при $\beta \in(0,1)$ выполняется неравенство

$$
\begin{aligned}
|\widetilde{v}|_{\beta} & \equiv\left(\int_{\Omega \cap \mathcal{U}} r^{2 \beta-2}\left(\left|\nabla_{x} \widetilde{v}(x)\right|^{2}+r^{-2}|\widetilde{v}(x)|^{2}\right) d x\right)^{1 / 2} \\
& \leqslant c_{p, \beta}\left(\left\|f ; L_{p}(\Omega)\right\|+\left\|\gamma ; W_{2}^{1}(\Gamma)\right\|\right) .
\end{aligned}
$$

2.4. Главный член внутреннего разложения (плоский пограничный слой). В криволинейных координатах $(s, y)$, где

$$
y=\left(y_{1}, y_{2}\right), \quad y_{1}=z, \quad y_{2}=n,
$$

лапласиан $\Delta_{x}$ записывается следующим образом

$$
\begin{aligned}
\Delta_{x} & =\mathscr{L}\left(s, y_{2}, \frac{\partial}{\partial s}, \frac{\partial}{\partial y}\right) \\
& =A(s, y)^{-1}\left(\frac{\partial}{\partial s} A(s, y)^{-1} \frac{\partial}{\partial s}+\frac{\partial}{\partial y_{2}} A(s, y) \frac{\partial}{\partial y_{2}}\right)+\frac{\partial^{2}}{\partial y_{1}^{2}} .
\end{aligned}
$$


В $(2.15) A(s, y)=1+y_{2} k(s)$ и $k(s)$ - кривизна контура Г в точке $s$. В плоскостях, перпендикулярных $\Gamma$, перейдем от $(2.14)$ к "быстрым" координатам

$$
\eta=\varepsilon^{-1} y, \quad \eta=\left(\eta_{1}, \eta_{2}\right) .
$$

Раскладывая коэффициенты $\mathscr{L}$ в ряды Тейлора по переменной $n=y_{2}=\varepsilon \eta_{2}$, получаем расщепление оператора (2.15)

$$
\Delta_{x} \sim \varepsilon^{-2} \Delta_{\eta}+\varepsilon^{-1} k(s) \partial_{2}+\varepsilon^{0}\left(\partial_{s}^{2}-\eta_{2} k(s)^{2} \partial_{2}\right)
$$

(явный вид младших членов не понадобится). В (2.17) и далее используются краткие обозначения $\partial_{s}=\partial / \partial s$ и $\partial_{i}=\partial / \partial \eta_{i}, i=1,2$.

Обратимся к соотношениям (1.2)-(1.5). Заменим в (1.2) “трехмерный” лапласиан $\Delta_{x}$ его расщеплением (2.17) и сохраним в последнем лишь старшее слагаемое - “двумерный" лапласиан $\Delta_{\eta}$ ("потеря" размерности вызвана тем, что переменная $s$ осталась "медленной" и дифференцирование $\partial_{s}$ несущественно при определении основного члена асимптотики в малой окрестности множества $\left.\Gamma_{\varepsilon}\right)$. Кроме того, при переходе к быстрым переменным уравнение (1.2) становится однородным: из-за множителя $\varepsilon^{-2}$ в $(2.17)$ следует пренебречь правой частью $f(x)=O(1)$. Ясно, что согласно (2.14), (2.16) $\partial_{n}=\varepsilon^{-1} \partial_{2}$, а потому необходимые изменения в (1.4) и (1.5) очевидны. Наконец, формула (1.1) для $\Gamma_{\varepsilon}$ принимает вид

$$
\Gamma_{\varepsilon}=\left\{(s, \eta): \eta_{1}=0, \eta_{2} \in \Upsilon(s) \equiv\left(-h_{-}(s), h_{+}(s)\right), s \in \Gamma\right\},
$$

и после предельного перехода к $\varepsilon=0$ множество $\mathscr{U} \cap \Omega$, записанное при помоши координат $(s, \eta)$, становится прямым произведением $\Gamma \times \mathbb{R}^{2}$. Все это позволяет заключить, что главньй член $e(\eta ; s)$ внутреннего разложения, обслуживаюшего малую окрестность полоски $\Gamma_{\varepsilon}$, обязан удовлетворять соотношениям

$$
\begin{gathered}
-\Delta_{\eta} e(\eta ; s)=0, \quad \eta \in \mathbb{R}_{+}^{2} \equiv\left\{\eta: \eta_{2}>0\right\} \\
\partial_{2} e\left(\eta_{1}, 0 ; s\right)=0, \quad \eta_{1} \in \mathbb{R} \backslash \overline{\Upsilon(s)} ; \\
e\left(\eta_{1}, 0 ; s\right) \geqslant \varphi(s), \quad \partial_{2} e\left(\eta_{1}, 0 ; s\right) \leqslant 0, \\
\left(e\left(\eta_{1}, 0 ; s\right)-\varphi(s)\right) \partial_{2} e\left(\eta_{1}, 0 ; s\right)=0, \quad \eta_{1} \in \Upsilon(s) .
\end{gathered}
$$

В рамках метода сращиваемых разложений следует дополнить формулы (2.18)-(2.20) некими требованиями, фиксируюшими поведение решения $e$ на бесконечности. Процедура сращивания подразумевает совпадение (формальное) главных членов асимптотик величин $v(x)$ и $e(\eta ; s)$ при $r \rightarrow 0$ и $\rho \equiv|\eta| \rightarrow \infty$, соответственно. Таким образом, в соответствии с (2.9), замыкаем вторую предельную задачу (2.18)-(2.20) следующим условием

$$
e(\eta ; s)=-\pi^{-1} \gamma(s) \ln (\varepsilon \rho)+J \gamma(s)+\psi(s)+o(1), \quad \rho \rightarrow \infty .
$$

Подчеркнем, что появление $\ln \varepsilon$ справа в (2.21) обусловлено заменой переменных $y \mapsto \eta$ и $r \mapsto \rho=\varepsilon^{-1} r$.

Ближайшая цель - построить явное решение задачи (2.18)-(2.21). Оказывается, что возможны только две крайние ситуации: всюду на отрезке $\Upsilon(s)=\left\{\eta: \eta_{1} \in \Upsilon(s), \eta_{2}=0\right\}$ реализуются либо условия Неймана, либо условия Дирихле. В первом случае согласно (2.18), (2.19) появляется однородная задача Неймана в полуплоскости $\mathbb{R}_{+}^{2}$, решением которой служит постоянная, а 
логарифмическая асимптотика решения невозможна (иными словами, $\gamma(s)=0$ ). Во втором случае решением получившейся смешанной краевой задачи также может выступать постоянная $\varphi(s)$ (напомним, что в (2.18)-(2.20) $s$ - произвольная, но фиксированная точка). Однако сушествуют решения смешанной задачи с логарифмическим ростом на бесконечности, условие (2.21) позволяет выбрать нужное решение (единственное). При этом $\gamma(s)>0$.

Реализуем намеченное. Непосредственно проверяется, что функция

$$
\eta \mapsto E(\eta ; s)=\frac{1}{\pi} \ln \left(\frac{2}{h(s)}\left|\zeta+\sqrt{\zeta^{2}-\left(\frac{h(s)}{2}\right)^{2}}\right|\right)
$$

где $\zeta=i \eta_{2}+\eta_{1}-\left(h_{+}(s)-h_{-}(s)\right) / 2, i=\sqrt{-1}, h(s)-$ внешний конформный радиус отрезка $\Upsilon(s)$ (см. [26]), удовлетворяет равенствам

$$
\begin{gathered}
-\Delta_{\eta} E(\eta ; s)=0, \quad \eta \in \mathbb{R}_{+}^{2} \\
E\left(\eta_{1}, 0 ; s\right)=0, \quad \eta_{1} \in \Upsilon(s) ; \quad \partial_{2} E\left(\eta_{1}, 0 ; s\right)=0, \quad \eta_{1} \in \mathbb{R} \backslash \overline{\Upsilon(s)} .
\end{gathered}
$$

Кроме того, для нее справедливы соотношения

$$
\begin{gathered}
E(\eta ; s)=\frac{1}{\pi} \ln \left(\frac{\rho}{H(s)}\right)+O\left(\rho^{-1}\right), \quad \rho \rightarrow \infty \\
E(\eta ; s)=\frac{1}{\pi} \sqrt{\frac{\rho_{ \pm}}{h(s)}} \sin \left(\frac{\theta_{ \pm}}{2}\right)+O\left(\rho_{ \pm}^{3 / 2}\right), \quad \rho_{ \pm} \rightarrow 0 \\
\partial_{2} E\left(\eta_{1}, 0 ; s\right)>0, \quad \eta_{1} \in \Upsilon(s) ; \quad E\left(\eta_{1}, 0 ; s\right)>0, \quad \eta_{1} \in \mathbb{R} \backslash \overline{\Upsilon(s)}
\end{gathered}
$$

в которых $\left(\rho_{ \pm}, \theta_{ \pm}\right)$- полярные координаты с центром $\left( \pm h_{ \pm}(s), 0\right) ; \theta_{ \pm} \in(0, \pi)$, $H(s)=(h(s)) / 4$.

ЗАмечАниЕ 2.2. В силу (2.23) функция (2.22), продолженная по четности на нижнюю полуплоскость $\left\{\eta: \eta_{2}<0\right\}$, оказывается решением однородной задачи Дирихле на плоскости с вырезанным отрезком $\Upsilon(s)$. Она носит название функции Грина с полюсом на бесконечности.

Положим

$$
e(\eta ; s)=-\gamma(s) E(\eta ; s)+a(s)
$$

где

$$
a(s)=-\pi^{-1} \gamma(s) \ln (\varepsilon H(s))+J \gamma(s)+\psi(s) .
$$

Благодаря $(2.23),(2.24)$ и (2.28), для суммы $(2.27)$ выполнены условия $(2.18),(2.19)$ и (2.21). Изучим возможность сохранения ограничений (2.20).

Если $\gamma(s)>0$, то в силу (2.26) $\partial_{2} e\left(\eta_{1}, 0 ; s\right)<0$ при $\eta_{1} \in \Upsilon(s)$. Следовательно, в первом случае из (2.20) должен появиться знак равенства (иначе неверно третье соотношение). Таким образом, на отрезке $\boldsymbol{\Upsilon}(s)$ поставлены условия Дирихле $e=\varphi$ и потому в силу $(2.23),(2.27)$ и $(2.28)$

$$
\gamma(s)>0 \Longrightarrow J \gamma(s)-\pi^{-1} \gamma(s) \ln (\varepsilon H(s))=\varphi(s)-\psi(s)
$$

Пусть теперь $\gamma(s)=0$. Тогда функция (2.27) становится постоянной, т.е. всюду на $\partial \mathbb{R}_{+}^{2}$ реализуются однородные условия Неймана. Первое неравенство в $(2.20)$ 
накладывает ограничение на величину (2.28): она должна быть не меньше $\varphi(s)$. В результате получаем, что

$$
\gamma(s)=0 \Longrightarrow J \gamma(s)-\pi^{-1} \gamma(s) \ln (\varepsilon H(s)) \geqslant \varphi(s)-\psi(s) .
$$

Наконец, при $\gamma(s)<0$ производная $\partial_{2}$ е положительна на $\boldsymbol{\Upsilon}(s)$, что противоречит второй формуле в (2.20). Значит, этот случай невозможен и возникает требование

$$
\gamma(s) \geqslant 0 \text {. }
$$

Соотношения (2.29)-(2.31) естественно рассматривать как задачу для определения плотности $\gamma$, фигурируюшей в (2.8).

2.5. Результирующее вариационное неравенство. Следуя общей схеме (см., например, [1]-[3]) соберем формулы (2.29)-(2.31) в единую задачу - вариационное неравенство. Умножим равенство в (2.29) и неравенство в (2.30) на произвольную неотрищательную функцию $\sigma \in C^{\infty}(\Gamma)$ и проинтегрируем по Г. Имеем

$$
(J \gamma, \sigma)_{\Gamma}-\pi^{-1}(\gamma \ln (\varepsilon H), \sigma)_{\Gamma} \geqslant(\varphi-\psi, \sigma)_{\Gamma} .
$$

Повторим процедуру с $\sigma=\gamma$. Из (2.29) и (2.30) следует формула

$$
(J \gamma, \gamma)_{\Gamma}-\pi^{-1}(\gamma \ln (\varepsilon H), \gamma)_{\Gamma}=(\varphi-\psi, \gamma)_{\Gamma}
$$

Вычтем (2.32) из (2.33) и получим

$$
(J \gamma, \gamma-\sigma)_{\Gamma}-\pi^{-1}(\gamma \ln (\varepsilon H), \gamma-\sigma)_{\Gamma} \leqslant(\varphi-\psi, \gamma-\sigma)_{\Gamma} \quad \forall \sigma \in C^{\infty}(\Gamma), \sigma \geqslant 0 .
$$

Результирующее вариационное неравенство формулируется как задача об отыскании неотрицательной функции $\gamma$, удовлетворяюшей соотношению $(2.34)$ при любой гладкой неотрицательной $\sigma$.

Отметим, что любое решение $\gamma$ задачи (2.34), обладаюшее некоторой гладкостью, подчиняется (2.29)-(2.31) (вывод этих формул из (2.34) также следует общей схеме [1]-[3]).

$\mathrm{K}$ сожалению, не удается доказать каких-либо утверждений о разрешимости задачи (2.34); причины поясняются ниже после перечисления свойств оператора (2.10). Однако для построения асимптотики решения исходной задачи (1.2)-(1.5) требуется только асимптотическое решение неравенства, т.е. плотность $\gamma_{*}$, удовлетворяюшая соотношениям (2.29)-(2.31) или (2.34) с малыми погрешностями. Это обстоятельство будет использоваться в следующих разделах.

Укажем некоторые свойства оператора $J$, обсуждавшиеся в [14], [17], [23], [24] и др. Ввиду симметричности ядер интегральных операторов в (2.10) J оказывается симметрическим и, кроме того, выполняется неравенство

$$
-(J \gamma, \gamma)_{\Gamma}+c(\gamma, \gamma)_{\Gamma} \geqslant \frac{1}{4 \pi}\left\{\int_{\Gamma} \int_{\Gamma} \frac{|\gamma(s)-\gamma(t)|^{2}}{|s-t|} d s d t+(\gamma, \gamma)_{\Gamma}\right\}
$$

с некоторой постоянной $c$. Согласно [27] выражение в фигурных скобках в (2.35) есть квадрат нормы функции $\gamma$ в пространстве Хёрмандера $H_{\mu}(\Gamma)$ с весовой функцией $\mu(\xi)=\max \left\{1,|\ln | \xi||^{1 / 2}\right\}$, норма в котором “склеивается" при помощи разбиения единицы из норм

$$
\left\|\gamma ; H_{\mu}(\mathbb{R})\right\|=\left(\int_{\mathbb{R}} \mu(\xi)^{2}|\mathscr{F} \gamma(\xi)|^{2} d \xi+\left\|\gamma ; L_{2}(\mathbb{R})\right\|^{2}\right)^{1 / 2}
$$


$\mathscr{F} \gamma$ - преобразование Фурье функции $\gamma$ (см. [28]). Кстати, весовой множитель $\mu(\xi)^{2} \sim \ln |\xi|$ в интеграле из $(2.36)$ вполне согласуется с тем, что $J-$ псевдодифференциальный оператор с главным символом $-\pi^{-1} \ln |\xi|$ (см., например, [29]).

Названные факты обусловливают постановку вариационного неравенства (2.34) на пространстве $H_{\mu}(\Gamma)$ : решение $\gamma$ и пробные функции $\sigma$ берутся из конуса $H_{\mu+}(\Gamma)=\left\{\tau \in H_{\mu}(\Gamma): \tau \geqslant 0\right\}$, а первое скалярное произведение слева понимается как двойственность между пространствами $H_{\mu}(\Gamma)^{*}$ и $H_{\mu}(\Gamma)$. Вместе с тем, оценка (2.35) и неравенство

$$
-\pi^{-1}(\gamma \ln (\varepsilon H), \gamma)_{\Gamma} \geqslant c|\ln \varepsilon|(\gamma, \gamma)_{\Gamma}
$$

верное при малом $\varepsilon>0$, означают, что оператор: $H_{\mu}(\Gamma) \rightarrow H_{\mu}(\Gamma)^{*}$, порожденный правой частью (2.34), оказывается суммой отрицательно и положительно определенных операторов, т.е. не имеет знака. Тем самым, применение классических результатов вьпуклого анализа (см. [1], [2] и др.) невозможно, и вопрос о разрешимости задачи (2.34) остается открытым.

Поясним на одном примере, почему задача (2.34) может не иметь точного решения. В случае $\varphi-\psi>0$ (см. п. 2.1.3 ${ }^{\circ}$ ) решение уравнения

$$
J \gamma(s)-\pi^{-1} \gamma(s) \ln (\varepsilon H)=\varphi(s)-\psi(s), \quad s \in \Gamma,
$$

оказывается положительным и потому удовлетворяет вариационному неравенству (2.34). Известна (см. [14], [24] и др.) основная особенность такого уравнения: сушествует бесконечно малая положительная последовательность $\left\{\varepsilon_{k}\right\}$ такая, что при $\varepsilon=\varepsilon_{k}$ уравнение (2.37), вообще говоря, неразрешимо (число $\pi^{-1} \ln \varepsilon_{k}$ является собственным для оператора $J-\pi^{-1} \ln H$ ). Вместе с тем, ввиду наличия большого положительного параметра $-\pi^{-1} \ln \varepsilon$ весьма логично предположить, что регулярное решение вариационного неравенства (2.34) положительно, а значит, удовлетворяет (2.37).

В [11], [14] предложены приемы построения асимптотических решений $\gamma_{*}$, оставляющих в $(2.37)$ невязку $O\left(\varepsilon^{T}\right)$ с произвольным, наперед заданным $T$. Однако перенесение их на случай вариационных неравенств затруднительно (дело в том, что в [11], [14] использовалась дополнительная гладкость правой части $\varphi-\psi$ и решения $\gamma_{*}$ уравнения (2.37), но она исчезает у решения результируюшего неравенства). В данной статье мы ограничиваемся конструкцией асимптотических решений задачи (2.34) с “логарифмической”, а не степенной точностью.

\section{§ 3. Главный член асимптотики и его обоснование}

3.1. Асимптотическое решение результирующего неравенства. Пусть, как и в $\S 2$, функции $f$ и $\varphi$ из (1.2) и (1.5) не зависят от $\varepsilon$. Примем еще предположение о множествах (2.5), сформулированное в п. 2.1.3 ${ }^{\circ}$ Задача (2.34) содержит большой параметр $\Lambda=|\ln \varepsilon|$ и естественно взять в качестве основного члена асимптотики ее решения функцию

$$
\gamma_{*}=\pi \Lambda^{-1}(\varphi-\psi)_{+},
$$

где $(t)_{+}=(t+|t|) / 2$ - положительная часть числа $t \in \mathbb{R}$. В самом деле, для $\gamma_{*}$, во-первых, выполнено условие (2.31) и, во-вторых, после замены $\gamma$ на $\gamma_{*}$ в левых частях равенства из (2.29) или неравенства из (2.30) появляется невязка $O\left(\Lambda^{-1}\right)$, меньшая по порядку, чем правые части $O(1)$ этих соотношений. 
Ясно, что $\gamma_{*} \in W_{\infty}^{1}(\Gamma)$. Заменим во всех формулах из $\S 2$ плотность $\gamma$ на $\gamma_{*}$. В силу предложения $2.1 .1^{\circ}$ решение (2.8) задачи (2.1) обладает асимптотикой (2.9), согласованной с асимптотикой главного члена (2.27) внутреннего разложения. Теперь, для того чтобы обосновать полученную асимптотику решения задачи (1.2)-(1.5), необходимо "склеить" внешнее и внутреннее разложения, определив глобальное асимптотическое приближение $U$, и оценить разность $u-U$.

Пусть $\chi \in C_{0}^{\infty}(\mathbb{R})$ - срезаюшая функция, равная единице в окрестности начала координат, $0 \leqslant \chi \leqslant 1$, и

$$
\chi_{\varepsilon}(x)=\chi\left(\varepsilon^{-1 / 2} r\right), \quad X_{\varepsilon}(x)=1-\chi\left(\varepsilon^{-1 / 2} r\right),
$$

причем $\chi_{\varepsilon}$ и $X_{\varepsilon}$ продолжены с $\mathscr{U} \cap \Omega$ на $\Omega$ нулем и единицей, соответственно. Положим

$$
U(\varepsilon, x)=X_{\varepsilon}(x) v_{*}(x)+\chi_{\varepsilon}(x)\left(-\gamma_{*}(s) E(\eta ; s)+a_{*}(s)\right),
$$

где координаты $(s, \eta)$ определены согласно (2.14) и (2.16), функция $v_{*}$ задана равенством (2.8) с заменой $\gamma$ на $\gamma_{*}$,

$$
a_{*}(s)=\pi^{-1} \Lambda \gamma_{*}(s)+\psi(s) .
$$

Отметим, что по сравнению с (2.28) из правой части (3.4) исчезла сумма $-\pi^{-1} \gamma_{*} \ln H+J \gamma_{*}$, представляюшая собой невязку, оставшуюся в соотношениях (2.29) и (2.30). Это обстоятельство снижает точность согласования внешнего и внутреннего разложений, но зато благодаря расположению носителей срезающих функций (3.2) становится верньм неравенство

$$
U(\varepsilon, x)=a_{*}(s)=(\varphi(s)-\psi(s))_{+}+\psi(s) \geqslant \varphi(s) \text { на } \Gamma_{\varepsilon} .
$$

Заметим, что $V \in W_{2}^{1}(\Omega)$, а интеграл из (2.8) - гладкая функция на $\operatorname{supp} X_{\varepsilon}$ (она имеет сингулярности только на $\Gamma)$. Кроме того, $\gamma_{*} \in W_{2}^{1}(\Gamma)$, а значит, и второе слагаемое из (3.3) попадает в пространство $W_{2}^{1}(\Omega)$. Наконец, $U=v_{*}=0$ на $\Sigma$, т.е. функция (3.3), рассматриваемая как глобальное приближение к решению задачи (1.2)-(1.5), содержится в множестве (1.6).

3.2. Конструкция глобального асимптотического приближения в общем случае. С целью распространить оценки на ситуации, с которыми мы столкнемся в следуюших параграфах, обобшим формулу (3.3).

Предположим, что справедливы представления

$$
f(\varepsilon, x)=f^{\circ}(\Lambda, x)+\widetilde{f}(\varepsilon, x), \quad \varphi(\varepsilon, s)=\varphi^{\circ}(\Lambda, s)+\widetilde{\varphi}(\varepsilon, s)
$$

с малыми остатками,

$$
\left\|\widetilde{f}(\varepsilon, \cdot) ; L_{2}(\Omega)\right\|=\delta_{f}, \quad\left\|\widetilde{\varphi}(\varepsilon, \cdot) ; W_{2}^{1}(\Gamma)\right\|=\delta_{\varphi} .
$$

Через $V(\Lambda, \cdot)$ обозначим ограниченное решение задачи $(2.1)$ с правой частью $f^{\circ}(\Lambda, \cdot) \in L_{p}(\Omega), p>3$, а через $\psi(\Lambda, \cdot) \in C^{1}(\Gamma)$ - его след на $Г$. Вид всех зависимостей от $\Lambda=|\ln \varepsilon|$ будет фиксирован позднее. Здесь для кратности не будем писать аргумент $\Lambda$ у функций $f^{\circ}, \varphi^{\circ}$ и т. д. Считаем, что $\varphi^{\circ} \in C^{1}(\Gamma)$. 
Допустим, что найдена неотрицательная функция $\gamma_{*} \in W_{2}^{1+\tau}(\Gamma)$, где $\tau \in\left(0, \frac{1}{2}\right)$, и справедливы высказывания

$$
\begin{aligned}
& \gamma_{*}(s)>0 \Longrightarrow J \gamma_{*}(s)-\pi^{-1} \gamma_{*}(s) \ln (\varepsilon H(s))-Y(s)=\varphi^{\circ}(s)-\psi(s) \\
& \gamma_{*}(s)=0 \Longrightarrow J \gamma_{*}(s)-\pi^{-1} \gamma_{*}(s) \ln (\varepsilon H(s))-Y(s) \geqslant \varphi^{\circ}(s)-\psi(s)
\end{aligned}
$$

Величина $Y$ играет роль невязки в соотношениях (2.29) и (2.30), ее малость выражается формулой, подобной (3.6),

$$
\left\|Y ; W_{2}^{1}(\Gamma)\right\|=\delta_{Y}
$$

Под $v_{*}$ подразумевается сумма $(2.8)$, где $\gamma=\gamma_{*}$. Пусть

$$
a_{*}(s)=\pi^{-1} \Lambda \gamma_{*}(s)+\psi(s)+J \gamma_{*}(s)-\pi^{-1} \gamma_{*}(s) \ln H(s)-Y(s)+\widetilde{\varphi}(\varepsilon, s)
$$

и $U$ - функция, заданная формулой (3.3) со всеми вышеуказанньми заменами. Как следует из (3.7) и (3.9), справедливо неравенство

$$
U(\varepsilon, x) \geqslant \varphi(\varepsilon, s), \quad x \in \Gamma_{\varepsilon}
$$

ЗАМЕчание 3.1 . В обозначениях, принятых в п. 3.1 , имеем $\widetilde{\varphi}=0$ и $\widetilde{f}=0$. Величины (3.4) и (3.9) совпадут, если положить $Y=-\pi^{-1} \gamma_{*} \ln H+J \gamma_{*}$; при этом $\delta_{Y}=O\left(\Lambda^{-1}\right)$ и, разумеется, $\delta_{\varphi}=\delta_{f}=0$.

Та же аргументация, что и в предыдущем разделе, позволяет заключить, что $U \in K$. Таким образом, можно подставить $U$ вместо $\zeta$ в (1.7) и получить, что

$$
\left(\nabla_{x} u, \nabla_{x} u-\nabla_{x} U\right)_{\Omega} \leqslant(f, u-U)_{\Omega} .
$$

В п. 3.3 будет выведено аналогичное неравенство, в котором $u$ и $U$ меняются местами, а в п. 3.4 на основе этих неравенств будет оценена норма $\left\|u-U ; W_{2}^{1}(\Omega)\right\|$.

3.3. Вариационное неравенство для $U$. В силу предположения о форме поверхности $\partial \Omega$ вблизи экватора $\Gamma$ (см. п. 1.1) и в силу определения (3.2) $\chi_{\varepsilon}$ и $X_{\varepsilon}$ функция $U$ удовлетворяет условию Неймана (1.4). Кроме того, используя неотрицательность плотности $\gamma_{*}$ и формулы $(2.23),(2.26)$, заключаем, что для $U$ выполняются соотношения (1.5). Так как $u \in K$, то теперь находим, что

$$
\begin{aligned}
& \left(\nabla_{x} U, \nabla_{x} U-\nabla_{x} u\right)_{\Omega}+\int_{\Omega}(U-u) \Delta_{x} U d x \\
& =-\int_{\Gamma_{\varepsilon}}(U-u) \partial_{n} U d s_{x}=-\int_{\Gamma_{\varepsilon}}(\varphi-u) \partial_{n} U d s_{x} \leqslant 0 .
\end{aligned}
$$

Преобразуем интеграл из левой части (3.12). С учетом (2.15) получаем

$$
\begin{aligned}
\Delta_{x} U=\left[\Delta_{x},\right. & \left.X_{\varepsilon}\right] v_{*}+X_{\varepsilon} \Delta_{x} v_{*}+\left[\Delta_{x}, \chi_{\varepsilon}\right]\left(a_{*}-\gamma_{*} E\right) \\
& +\chi_{\varepsilon} A^{-1} \frac{\partial}{\partial s} A^{-1} \frac{\partial}{\partial s}\left(a_{*}-\gamma_{*} E\right)-\chi_{\varepsilon} A^{-1} \frac{\partial A}{\partial y_{2}} \gamma_{*} \frac{\partial E}{\partial y_{2}}-\chi_{\varepsilon} \gamma_{*} \Delta_{y} E .
\end{aligned}
$$


Здесь $[\mathscr{A}, \mathscr{B}]=\mathscr{A} \mathscr{B}-\mathscr{B} \mathscr{A}$-коммутатор операторов $\mathscr{A}$ и $\mathscr{B}$, а функции имеют те же аргументы, что и в $(3.3),(2.15)$. Вспоминая уравнения, которым удовлетворяют $v_{*}$ и $E$, и обрашаясь к $(3.2),(3.5)$, продолжаем преобразования

$$
\begin{aligned}
-\Delta_{x} U= & f-\tilde{f}-\chi_{\varepsilon} f^{\circ}+\left[\Delta_{x}, \chi_{\varepsilon}\right]\left(v_{*}-a_{*}+\gamma_{*} E\right) \\
& +\chi_{\varepsilon} A^{-1} \frac{\partial A}{\partial y_{2}} \gamma_{*} \frac{\partial E}{\partial y_{2}}-\chi_{\varepsilon} A^{-1} \frac{\partial}{\partial s} A^{-1} \frac{\partial}{\partial s}\left(a_{*}-\gamma_{*} E\right) \\
\equiv & f+F_{1}+F_{2}+F_{3}+F_{4}+F_{5} .
\end{aligned}
$$

Итак,

$$
\left(\nabla_{x} U, \nabla_{x} U-\nabla_{x} u\right)_{\Omega} \leqslant(f, U-u)_{\Omega}+\sum_{m=1}^{5}\left(F_{m}, U-u\right)_{\Omega} .
$$

Сложим неравенства (3.11) и (3.14). Имеем

$$
\left\|\nabla_{x} u-\nabla_{x} U ; L_{2}(\Omega)\right\|^{2} \leqslant \sum_{m=1}^{5}\left(F_{m}, U-u\right)_{\Omega} .
$$

Далее норму разности $\nabla_{x} u-\nabla_{x} U$ в $L_{2}(\Omega)$ обозначаем $M$.

3.4. Оценка правой части неравенства (3.15). Приступим к оценке скалярных произведений $S_{m}=\left(F_{m}, U-u\right)_{\Omega}$. Будем рассматривать их в порядке возрастания сложности вывода оценки.

Сначала обратимся к случаю $m=5$. Интегрируя по частям в криволинейных координатах $(s, y)$, получим

$$
\begin{aligned}
\left|S_{5}\right| & =\left|\int_{\Gamma} \int_{\mathbb{R}_{+}^{2}} \chi_{\varepsilon} A^{-1} \frac{\partial}{\partial s}(U-u) \frac{\partial}{\partial s}\left(a_{*}-\gamma_{*} E\right) d s d y\right| \\
& \leqslant c M\left\{\int_{\Gamma} \int_{\mathbb{R}_{+}^{2}} \chi_{\varepsilon}\left|\frac{\partial a_{*}}{\partial s}\right|^{2} d s d y+\int_{\Gamma} \int_{\mathbb{R}_{+}^{2}} \chi_{\varepsilon}\left|\frac{\partial}{\partial s} \gamma_{*} E\right|^{2} d s d y\right\}^{1 / 2} .
\end{aligned}
$$

Подчеркнем, что $C>A>c>0$ в $\mathscr{U}$ и поверхностные интегралы не возникают. Так как $\chi_{\varepsilon}(x)=0$ при $r>c \varepsilon^{1 / 2}$, то в силу (3.9) для первого интеграла $I_{1}$ в фигурных скобках в (3.16) верно неравенство

$$
\left|I_{1}\right| \leqslant c \varepsilon\left(\left(1+\Lambda^{2}\right)\left\|\gamma_{*} ; W_{2}^{1}(\Gamma)\right\|^{2}+\left\|\psi ; W_{2}^{1}(\Gamma)\right\|^{2}+\left\|J \gamma_{*} ; W_{2}^{1}(\Gamma)\right\|^{2}+\delta_{Y}^{2}+\delta_{\varphi}^{2}\right) .
$$

В начале п. 2.1 уже упоминалось, что

$$
\left\|\psi ; W_{2}^{1}(\Gamma)\right\| \leqslant c_{p}\left\|f^{\circ} ; L_{p}(\Omega)\right\| \equiv c_{p} \delta_{o}, \quad p>3 .
$$

Кроме того, ввиду свойств оператора $J$, перечисленных в п. 2.4,

$$
\left\|J \gamma_{*} ; W_{2}^{1}(\Gamma)\right\| \leqslant c_{\tau}\left\|\gamma_{*} ; W_{2}^{1+\tau}(\Gamma)\right\| \equiv c_{\tau} \delta_{\gamma}, \quad \tau \in\left(0, \frac{1}{2}\right) .
$$

Следовательно, при некоторых фиксированных $\tau$ и $p$

$$
\left|I_{1}\right|^{1 / 2} \leqslant c \varepsilon^{1 / 2}\left(\delta_{Y}+\delta_{\varphi}+\delta_{o}+(1+\Lambda) \delta_{\gamma}\right) .
$$


Рассмотрим последний интеграл $I_{2}$ в (3.16). Заметим, что согласно (2.22)

$$
E(\eta ; t)=E\left(\frac{h(s)}{h(t)}\left(\eta_{1}-h_{+}(s)\right)+h_{+}(t), \frac{h(s)}{h(t)} \eta_{2} ; s\right)-\ln \frac{h(t)}{h(s)} .
$$

Поэтому, учитывая (2.24) и (2.25), находим

$$
\begin{gathered}
\int_{|y| \leqslant c \varepsilon^{1 / 2}}\left|E\left(\varepsilon^{-1} y ; s\right)\right|^{2} d y \leqslant c \varepsilon\left(1+|\ln \varepsilon|^{2}\right), \\
\int_{|y|<c \varepsilon^{1 / 2}}\left|\partial_{s} E\left(\varepsilon^{-1} y ; s\right)\right|^{2} d y \leqslant c \int_{|y|<c \varepsilon^{1 / 2}}\left(1+\varepsilon^{-2}|y|^{2}\right)\left|\nabla_{\eta} E\left(\varepsilon^{-1} y ; s\right)\right|^{2} d y \leqslant c \varepsilon .
\end{gathered}
$$

Отсюда выводим неравенство

$$
\left|I_{2}\right|^{1 / 2} \leqslant c \varepsilon^{1 / 2}(1+\Lambda)\left\|\gamma_{*} ; W_{2}^{1}(\Gamma)\right\|,
$$

которое вместе с (3.19) дает искомую оценку

$$
\left|S_{5}\right| \leqslant c \varepsilon^{1 / 2} M\left(\delta_{Y}+\delta_{\varphi}+\delta_{o}+(1+\Lambda) \delta_{\gamma}\right) .
$$

Следуюшее утверждение вытекает, например, из принципа Рэлея.

Лемма 3.2. Для функции $R \in W_{2}^{1}(\Omega)$, удовлетворяющей условию $R=0$ на $\Sigma$, справедливо неравенство

$$
\left\|\nabla_{x} R ; L_{2}(\Omega)\right\| \geqslant \lambda^{1 / 2}\left\|R ; L_{2}(\Omega)\right\|,
$$

где $\lambda$ - первое собственное число задачи (2.1), $\lambda>0$.

Теперь нетрудно оценить скалярное произведение, содержашее $\widetilde{f}$,

$$
\left|S_{1}\right| \leqslant\left\|\widetilde{f} ; L_{2}(\Omega)\right\| \cdot\left\|U-u ; L_{2}(\Omega)\right\| \leqslant \lambda^{-1 / 2} \delta_{f} M .
$$

ЛЕмма 3.3. В условиях леммы 3.2 выполняется неравенство

$$
\left\|\nabla_{x} R ; L_{2}(\Omega)\right\| \geqslant c\left\|r^{-1}(1+|\ln r|)^{-1} R ; L_{2}(\Omega)\right\| .
$$

ДоКАЗАТЕЛЬСТво. На множестве $\Omega \backslash \mathscr{U}$ весовой множитель из правой части (3.23) отделен от нуля и ограничен. Кроме того, в силу леммы 3.2

$$
\left\|\nabla_{x}\left(\chi_{d} R\right) ; L_{2}(\Omega)\right\| \leqslant c_{d}\left\|\nabla_{x} R ; L_{2}(\Omega)\right\|,
$$

здесь $d$ малое, но фиксированное положительное число. Таким образом, достаточно оценить интеграл

$$
\int_{\Omega \cap \mathscr{U}} r^{-2}(1+|\ln r|)^{-2}\left|\chi_{d}(x) R(x)\right|^{2} d x .
$$

Это делается при помощи известного одномерного неравенства Харди

$$
\int_{0}^{2 d} r^{-1}\left|\ln \frac{r}{2 d}\right|^{-2}|Z(r)|^{2} d r<4 \int_{0}^{2 d} r\left|\frac{d Z}{d r}(r)\right|^{2} d r
$$


справедливого для любой функции $Z \in C_{0}^{\infty}[0,2 d)$, которое нужно дополнительно проинтегрировать по длине дуги $s \in$ Г и полярному углу $\theta \in[0, \pi)$, учесть формулу для якобиана при замене $(s, r, \theta) \mapsto(s, y) \mapsto x$ и замкнуть полученное соотношение по норме в $W_{2}^{1}(\Omega)$.

Теперь доступны для рассмотрения второй и четвертый члены справа в (3.15). Имеем

$$
\begin{aligned}
\left|S_{2}\right| & \leqslant\left\|r^{-1}(1+|\ln r|)^{-1}(u-U) ; L_{2}(\Omega)\right\| \cdot\left\|r(1+|\ln r|) \chi_{\varepsilon} f^{\circ} ; L_{2}(\Omega)\right\| \\
& \leqslant c M \varepsilon^{1 / 2}(1+|\ln \varepsilon|)\left\|f^{\circ} ; L_{2}(\Omega)\right\| \leqslant c \varepsilon^{1 / 2}(1+\Lambda) \delta_{o} M \\
\left|S_{4}\right| & \leqslant c M\left(\int_{\Gamma}\left|\gamma_{*}(s)\right|^{2} \int_{|y| \leqslant c \varepsilon^{1 / 2}} r^{2}(1+|\ln r|)^{2}\left|\frac{\partial E}{\partial y_{2}}\left(\frac{y}{\varepsilon}, s\right)\right|^{2} d s d y\right)^{1 / 2} \\
& \leqslant c M\left\|\gamma_{*} ; L_{2}(\Gamma)\right\| \varepsilon^{1 / 2}(1+|\ln \varepsilon|) \leqslant c \varepsilon^{1 / 2}(1+\Lambda) \delta_{\gamma} M
\end{aligned}
$$

В (3.24) принята во внимание узость носителя $\chi_{\varepsilon}$, а в $(3.25)$ использована подобная (3.20) оценка для интеграла, содержащего $\nabla_{y} E$.

Наибольшие сложности доставляет оценка выражения $S_{3}$, поскольку неравенство (3.23) оказывается слишком грубым для него из-за логарифмического множителя. Сначала отщепим от $S_{3}$ те интегралы, для изучения которых достаточно применить неравенство (3.23). Замечая, что коэффициенты коммутатора $\left[\Delta_{x}, \chi_{\varepsilon}\right]$ аннулируются вне множества $T \times \Gamma=\left\{x \in \mathscr{U} \cap \Omega: c \varepsilon^{1 / 2} \leqslant r \leqslant C \varepsilon^{1 / 2}\right\}$ и обращаясь к формуле (2.13), получаем, что при $\gamma_{*} \in W_{2}^{1}(\Gamma)$

$$
\begin{aligned}
& \left|\int_{\Omega}(U-u)\left[\Delta_{x}, \chi_{\varepsilon}\right] \widetilde{v}_{*} d x\right| \\
& \quad \leqslant c M\left(\int_{T \times \Gamma} r^{2}(1+|\ln r|)^{2}\left(\varepsilon^{-2}\left|\widetilde{v}_{*}(x)\right|^{2}+\varepsilon^{-1}\left|\nabla_{x} \widetilde{v}_{*}(x)\right|^{2}\right) d x\right)^{1 / 2} \\
& \quad \leqslant c M\left(\int_{T \times \Gamma}(1+|\ln r|)^{2}\left(r^{-2}\left|\widetilde{v}_{*}(x)\right|^{2}+\left|\nabla_{x} \widetilde{v}_{*}(x)\right|^{2}\right) d x\right)^{1 / 2} \\
& \quad \leqslant c_{\beta} M(1+|\ln \varepsilon|) \varepsilon^{(1-\beta) / 2}\left|\widetilde{v}_{*}\right|_{\beta} \leqslant c_{p, \beta} \varepsilon^{(1-\beta) / 2}(1+\Lambda)\left(\delta_{o}+\delta_{\gamma}\right) M
\end{aligned}
$$

Здесь функция $\widetilde{v}_{*}$ определена равенством (2.11) с обычными заменами. Далее выбираем $\beta=1 / 2$ - некоторое огрубление этой оценки не скажется на окончательных результатах.

ЗАмЕчАнИЕ 3.4. Если $\gamma_{*} \in W_{\infty}^{1}(\Gamma)$, то можно применить неравенство $(2.12)$ из предложения $2.1 .1^{\circ}$

$$
\begin{aligned}
\left|\int_{\Omega}(U-u)\left[\Delta_{x}, \chi_{\varepsilon}\right] \widetilde{v}_{*} d x\right| & \leqslant c M\left(\left\|f ; L_{p}(\Omega)\right\|+\left\|\gamma_{*} ; W_{\infty}^{1}(\Gamma)\right\|\right)\left(\int_{T}(1+|\ln r|)^{4} d r\right)^{1 / 2} \\
& \leqslant c \varepsilon^{1 / 2}(1+\Lambda)^{2}\left(\delta_{o}+\left\|\gamma_{*} ; W_{\infty}^{1}(\Gamma)\right\|\right) M .
\end{aligned}
$$

Точно так же из представления (2.24) вьводим

$$
\begin{aligned}
& \left|\int_{\Omega}(U-u)\left[\Delta_{x}, \chi_{\varepsilon}\right]\left(\gamma_{*}\left(E-\pi^{-1} \ln \left(\frac{\rho}{H}\right)\right)\right) d x\right| \\
& \leqslant c M\left(\int_{T \times \Gamma} r^{2}(1+|\ln r|)^{2}\left(\varepsilon^{-2} \rho^{-4}+\varepsilon^{-1} \rho^{-2}\right)\left|\gamma_{*}(s)\right|^{2} d x\right)^{1 / 2} \\
& \leqslant c M \varepsilon^{1 / 2}(1+|\ln \varepsilon|)\left\|\gamma_{*} ; L_{2}(\Gamma)\right\| \leqslant c \varepsilon^{1 / 2}(1+\Lambda) \delta_{\gamma} M .
\end{aligned}
$$


Обрашаясь к $(2.9),(2.24)$ и (3.9), видим, что осталось оценить интеграл

$$
I=\int_{\Omega}(U-u)\left[\Delta_{x}, \chi_{\varepsilon}\right](Y-\widetilde{\varphi}) d x .
$$

Функции $Y$ и $\widetilde{\varphi}$ зависят от переменной $s$, а функция $\chi_{\varepsilon}-$ от $r$ (т.е. от $y$ ). Поэтому в силу $(2.15)$

$$
\begin{aligned}
& {\left[\Delta_{x}, \chi\left(\varepsilon^{-1 / 2} r\right)\right](Y(s)-\widetilde{\varphi}(s))=(Y(s)-\widetilde{\varphi}(s))\left(A\left(s, y_{2}\right)^{-1} \Delta_{y} \chi\left(\varepsilon^{-1 / 2}|y|\right)\right.} \\
& \left.+\left(1-A\left(s, y_{2}\right)^{-1}\right) \Delta_{y} \chi\left(\varepsilon^{-1 / 2}|y|\right)+A\left(s, y_{2}\right)^{-1} \frac{\partial A}{\partial y_{2}}(s, y) \frac{\partial}{\partial y_{2}} \chi\left(\varepsilon^{-1 / 2}|y|\right)\right) .
\end{aligned}
$$

В соответствии с разбиением (3.26) представим $I$ как сумму $I^{(1)}+I^{(2)}+I^{(3)}$. Поскольку $A\left(s, y_{2}\right)=1+y_{2} k(s)$ и $\left|\nabla_{y}^{k} \chi\left(\varepsilon^{-1 / 2}|y|\right)\right| \leqslant c_{k} \varepsilon^{-k / 2}$, то в силу (3.23)

$$
\begin{aligned}
\left|I^{(2)}\right| & \leqslant c M\left(\int_{\Gamma}|Y(s)-\widetilde{\varphi}(s)|^{2} d s\right)^{1 / 2}\left(\varepsilon^{-2} \int_{T} r^{2}(1+|\ln r|)^{2}\left|y_{2}\right|^{2} d y\right)^{1 / 2} \\
& \leqslant c \varepsilon^{1 / 2}(1+\Lambda)\left(\delta_{Y}+\delta_{\varphi}\right) M
\end{aligned}
$$

Аналогично,

$$
\begin{aligned}
\left|I^{(3)}\right| & \leqslant c M\left(\int_{\Gamma}|Y(s)-\widetilde{\varphi}(s)|^{2} d s\right)^{1 / 2}\left(\varepsilon^{-1} \int_{T} r^{2}(1+|\ln r|)^{2} d y\right)^{1 / 2} \\
& \leqslant c \varepsilon^{1 / 2}(1+\Lambda)\left(\delta_{Y}+\delta_{\varphi}\right) M .
\end{aligned}
$$

Наконец, в интеграле $I^{(1)}$ перейдем к криволинейным координатам $(s, y)$ (соответствуюший якобиан равен $\left.A\left(s, y_{2}\right)\right)$. Имеем

$$
I^{(1)}=\int_{\Gamma} \int_{\mathbb{R}_{+}^{2}}(U(\varepsilon, x)-u(\varepsilon, x))(Y(s)-\widetilde{\varphi}(s)) \Delta_{y} \chi\left(\varepsilon^{-1 / 2}|y|\right) d s d y
$$

Именно для этого интеграла применение оценки (3.23) влечет нежелательную погрешность.

Проведем вспомогательные построения. Положим $R(s, y)=U(\varepsilon, x)-u(\varepsilon, x)$ и

$$
\bar{R}(s)=\left(\operatorname{mes}_{2} T\right)^{-1} \int_{T} R(s, y) d y, \quad \widetilde{R}(s, y)=R(s, y)-\bar{R}(s) .
$$

По неравенству Пуанкаре

$$
\int_{\Gamma}\left(\int_{T}\left|\nabla_{y} \widetilde{R}(s, y)\right|^{2} d y\right) d s \geqslant c \varepsilon^{-1} \int_{\Gamma}\left(\int_{T}|\widetilde{R}(s, y)|^{2} d y\right) d s
$$

здесь множитель $\varepsilon^{-1}=\left(\varepsilon^{1 / 2}\right)^{-2}$ учитывает зависимость константы от размеров области $T=\left\{y \in \mathbb{R}^{2}: C \varepsilon^{1 / 2} \geqslant|y| \geqslant c \varepsilon^{1 / 2}, y_{2}>0\right\}$.

Согласно определению срезаюшей функции (см. п. 3.1)

$$
\int_{T} \Delta_{y} \chi\left(\varepsilon^{-1 / 2}|y|\right) d y=0, \quad \operatorname{supp} \Delta_{y} \chi_{\varepsilon}=T .
$$


Следовательно, в (3.27) разность $U-u$ можно заменить на $\widetilde{R}$. Тогда в силу (3.28)

$$
\begin{aligned}
\left|I^{(1)}\right| \leqslant c\left(\int_{\Gamma} \int_{T}\left|\nabla_{y} \widetilde{R}(s, y)\right|^{2} d s d y\right)^{1 / 2} & \\
& \times\left(\int_{\Gamma}|Y(s)-\widetilde{\varphi}(s)|^{2} d s \int_{T} \varepsilon\left|\Delta_{y} \chi\left(\varepsilon^{-1 / 2}|y|\right)\right|^{2} d y\right)^{1 / 2} .
\end{aligned}
$$

Осталось обратить внимание на очевидное равенство $\nabla_{y} \widetilde{R}=\nabla_{y} U-\nabla_{y} u$ и получить

$$
\left|I^{(1)}\right| \leqslant c\left(\delta_{Y}+\delta_{\varphi}\right) M
$$

Итак, оценен последний фрагмент выражения $S_{3}$. Используя полученные неравенства и учитывая малость параметра $\varepsilon>0$, заключаем, что

$$
\left|S_{3}\right| \leqslant c\left(\delta_{Y}+\delta_{\varphi}+\varepsilon^{1 / 4}(1+\Lambda)\left(\delta_{o}+\delta_{\gamma}\right)\right) M
$$

Теперь, учитывая формулы (3.21), (3.22), (3.24), (3.25), (3.29), находим мажоранту для правой части (3.15).

ТЕОРема 3.5. Пусть выполнены требования, перечисленнье в n. 3.2. Тогда решение и задачи (1.2)-(1.5) и функиия (3.3), определенная по плотности $\gamma_{*}$ из (3.7), связаны соотношением

$$
\left\|\nabla_{x} u-\nabla_{x} U ; L_{2}(\Omega)\right\| \leqslant c\left(\delta_{f}+\delta_{\varphi}+\delta_{Y}+\varepsilon^{1 / 4}(1+|\ln \varepsilon|)\left(\delta_{o}+\delta_{\gamma}\right)\right),
$$

в котором $\delta$-константы взяты из (3.6), (3.8), (3.17), (3.18).

3.5. Обоснование главного члена асимптотики. Согласно замечанию 3.1 в ситуации, рассмотренной в $\S 2$ и в п. 3.1 , имеем $\delta_{f}=\delta_{\varphi}=0$ и $\delta_{Y}=O\left(\Lambda^{-1}\right)$. Кроме того, $\delta_{o}=O(1)$ и $\delta_{\gamma}=O\left(\Lambda^{-1}\right)$ в соответствии с $(3.17),(3.18)$ и $(3.1)$. Теперь можно конкретизировать теорему 3.5 .

Теорема 3.6. Пусть справедливы предположения, сформулированные в n. 2.1 и в n. 3.1. Пусть еще $U$ - глобальное асимптотическое приближение (3.3) к решению и задачи (1.2)-(1.5), причем $\gamma_{*}-$ плотность (3.1), $v_{*}$ решение (2.8) задачи (2.1) при $\gamma=\gamma_{*}, E-$ функиия (2.22), $a_{*}$ - величина (3.4), а $X_{\varepsilon} и \chi_{\varepsilon}-$ срезающие функиии (3.2). Тогда справедливо неравенство

$$
\left\|\nabla_{x} u-\nabla_{x} U ; L_{2}(\Omega)\right\| \leqslant c|\ln \varepsilon|^{-1},
$$

в котором постоянная с не зависит от $\varepsilon \in\left(0, \varepsilon_{0}\right), \varepsilon_{0}>0$.

Приведем несколько комментариев к теореме 3.6 .

В (3.31) содержится только оценка для нормы градиента, но, разумеется, леммы 3.2 и 3.3 дают оценки и самой разности $u-U$. Сказанное в равной мере относится и к неравенству (3.30).

Нетрудно проверить соотношения

$$
\begin{gathered}
\left\|X_{\varepsilon} V+\chi_{\varepsilon} \psi ; W_{2}^{1}(\Omega)\right\|=O(1), \\
\left\|X_{\varepsilon}\left(v_{*}-V\right) ; W_{2}^{1}(\Omega)\right\|=O(1), \\
\left\|\chi_{\varepsilon}\left(a_{*}-\gamma_{*} E-\psi\right) ; W_{2}^{1}(\Omega)\right\|=O(1) .
\end{gathered}
$$


Таким образом, из (3.31) вытекает, в частности, что каждое из слагаемых, указанных в (3.32), включено в глобальное асимптотическое приближение $U(\varepsilon, x)$ обосновано. Это обстоятельство, конечно же, оправдывает асимптотические построения, проведенные в $\S 2$.

В п. 2.1.3 к функциям $\psi$ и $\varphi$ предъявлены завышенные требования. Просматривая доказательство теоремы 3.5, убеждаемся в том, что теорема 3.5 сохраняет силу, если $(\varphi-\psi)_{+} \in W_{2}^{1+\tau}(\Gamma)$ при каком-нибудь $\tau \in\left(0, \frac{1}{2}\right)$. Тем не менее, условие $\varphi$, $\psi \in C^{1}(\Gamma)$ позволяет использовать более простое утверждение 1) предложения 2.1 (см. замечание 3.4 ).

Несмотря на то, что найдена асимптотика решения $u$, достоверная информация о зоне контакта неизвестна. Конечно, весьма правдоподобен тот факт, что эта зона мало отличается от $\left\{x \in \Gamma_{\varepsilon}: \gamma_{*}(s)>0\right\}$, однако обосновать его не удается. Дело в том, что утверждение теоремы 3.6 недостаточно для оценки (поточечной) следа разности $u-U$ на $\Gamma_{\varepsilon}$.

ЗАмЕчАнИЕ 3.7. В случае $\psi<\varphi$ (т.е. $\gamma_{*}>0$; ср. с п. 2.1.2 ${ }^{\circ}$ ) имеется подход, позволяющий установить, что экваториальная полоска (1.1) занята областью контакта целиком. Он был реализован в работе [9], посвященной задаче Синьорини с препятствиями на множествах с мальми диаметрами. Центральный момент - проверка совпадения решений нелинейной задачи (1.2)-(1.5) и линейной задачи (1.2)-(1.4), (2.6). Соответствующее доказательство использует асимптотически точные весовые оценки решений краевых задач в областях с сингулярно возмущенной границей (см. [30], [24] и др.). Оно весьма громоздко и в настоящей статье, как и в [4]-[8], мы ограничиваемся формальными рассуждениями о структуре зоны контакта. Подчеркнем, что в наиболее интересном случае (разность $\varphi-\psi$ меняет знак) упомянутый подход все равно не годится из-за полного отсутствия сведений о строении зоны контакта вблизи точек множества $\Xi^{0}$ (см. п. 2.1.3 $)$.

\section{§4. Второй член асимптотики решения. Вариация зоны контакта}

4.1. Двучленная асимптотика решения результируюшего неравенства (попытка первая). Пусть функции $f$ и $\varphi$ из (1.2) и (1.5) допускают представления

$$
\begin{aligned}
& f(\varepsilon, x)=f_{0}(x)+\Lambda^{-1} f_{1}(x)+\widetilde{f}(\varepsilon, x), \\
& \varphi(\varepsilon, s)=\varphi_{0}(s)+\Lambda^{-1} \varphi_{1}(s)+\widetilde{\varphi}(\varepsilon, s),
\end{aligned}
$$

причем $f_{i} \in L_{p}(\Omega)$ при $p>3, \varphi_{i} \in C^{1}(\Gamma)$ и в соответствии с (3.6)

$$
\delta_{f}=O\left(\Lambda^{-2}\right), \quad \delta_{\varphi}=O\left(\Lambda^{-2}\right) .
$$

Для $i=0,1$ найдем ограниченные решения $V_{i}$ задачи $(2.1)$ с правьми частями $f_{i}$; через $\psi_{i} \in C^{1}(\Gamma)$ обозначим следы $V_{i}$ на $\Gamma$. Плотность $\widehat{\gamma}$ и сингулярное решение $\widehat{v}$ задачи (2.1) с правой частью $f^{\circ}=f_{0}+\Lambda^{-1} f_{1}$ попробуем искать в виде

$$
\begin{aligned}
& \widehat{\gamma}(s)=\Lambda^{-1} \gamma_{0}(s)+\Lambda^{-2} \gamma_{1}(s), \\
& \widehat{v}(x)=V_{0}(x)+\Lambda^{-1} V_{1}(x)+\int_{\Gamma} \widehat{\gamma}(t) G(x ; t) d t .
\end{aligned}
$$

Заметим, что из формул (4.3) и (4.4) можно отбросить слагаемые с нижними индексами 1 и сохранить утверждение теоремы 3.6 (при выводе оценки (3.31) из (3.30) в мажоранте появится дополнительная величина

$$
c\left(\left\|f-f_{0} ; L_{2}(\Omega)\right\|+\left\|\varphi-\varphi_{0} ; W_{2}^{1}(\Gamma)\right\|\right),
$$


которая согласно (4.1) и (4.2) есть $\left.O\left(|\ln \varepsilon|^{-1}\right)\right)$. Таким образом, нам известно первое слагаемое справа в (4.3)

$$
\gamma_{0}(s)=\pi\left(\varphi_{0}(s)-\psi_{0}(s)\right)_{+}
$$

(см. (3.1)). Попытаемся построить асимптотическую поправку $\Lambda^{-2} \gamma_{1}$. Если бы вместо вариационного неравенства

$$
\begin{gathered}
(J \gamma, \gamma-\sigma)_{\Gamma}-\pi^{-1}(\gamma \ln (\varepsilon H), \gamma-\sigma)_{\Gamma} \leqslant\left(\varphi_{0}-\psi_{0}+\Lambda^{-1}\left(\varphi_{1}-\psi_{1}\right), \gamma-\sigma\right)_{\Gamma} \\
\forall \sigma \in C^{\infty}(\Gamma), \quad \sigma \geqslant 0,
\end{gathered}
$$

(ср. с (2.34)) мы решали уравнение

$$
J \gamma(s)-\pi^{-1} \gamma(s) \ln (\varepsilon H(s))=\varphi_{0}(s)-\psi_{0}(s)+\Lambda^{-1}\left(\varphi_{1}(s)-\psi_{1}(s)\right), \quad s \in \Gamma
$$

(ср. с (2.37)), то, подставляя (4.3) в (4.7) и собирая коэффициенты при одинаковых степенях $\Lambda$, мы пришли бы к формуле

$$
\gamma_{1}(s)=\gamma_{0}(s) \ln H(s)-\pi J \gamma_{0}(s)+\pi\left(\varphi_{1}(s)-\psi_{1}(s)\right)
$$

Однако необходимо учесть условие (2.31) неотрицательности плотности. Явно недостаточно в качестве $\gamma_{1}$ взять положительную часть выражения (4.8): оно может оказаться меньше нуля в тех точках $s$, для которых $\gamma_{0}(s)>0$ и отрицательные значения $\gamma_{1}(s)$ допустимы при большом $\Lambda=|\ln \varepsilon|$. Итак, простые представления (4.3) и (4.4) для асимптотического решения непригодны.

4.2. Вариация зоны контакта. Как уже говорилось в конце $\S 3$, в рамках формального асимптотического анализа множество $\Gamma_{\varepsilon}^{+}=\left\{x \in \Gamma_{\varepsilon}: s \in \Xi^{+}\right\}$ выступает как главное приближение к зоне контакта $\Xi^{+}(\varepsilon)$ (обозначения см. в п. 2.1 ; при этом $\varphi, \psi$ заменяются на $\left.\varphi_{0}, \psi_{0}\right)$. Для тех $s \in \Xi^{+}$, для которых $\gamma_{0}(s) \equiv \varphi_{0}(s)-\psi_{0}(s)>c_{0}>0$, величина $\widehat{\gamma}(s)$ вычисляется по формуле (4.3), тогда условие (2.31) не нарушается (при достаточно малом $\varepsilon$ ), а из уравнения (4.7) будет устранена невязка $O\left(\Lambda^{-1}\right)$. Если же $s \in \Xi^{-}$и $\varphi_{0}(s)-\psi_{0}(s)<-c_{0}<0$, то положим $\gamma_{1}(s)=0$ и $\widehat{\gamma}(s)=0$ : в таких точках неравенство

$$
J \gamma(s)-\pi^{-1} \gamma(s) \ln (\varepsilon H(s))>\varphi_{0}(s)-\psi_{0}(s)+\Lambda^{-1}\left(\varphi_{1}(s)-\psi_{1}(s)\right)
$$

(cp. с (2.30)) для $\widehat{\gamma}$, по-прежнему, выполнено. Таким образом, представление для $\widehat{\gamma}$ не выяснено только в малой окрестности множества $\Xi^{0}$, где, как будет видно из дальнейшего, происходит возмушение множества $\Gamma_{\varepsilon}^{+}$, описьвающего зону контакта.

Пусть $\mathscr{V}_{q} \subset \Gamma$ - малая окрестность точки $s_{q} \in \Xi^{0}$ и, для определенности, $\left\{s \in \mathscr{V}_{q}: \pm s> \pm s_{q}\right\} \subset \Xi^{ \pm}\left(\right.$см. предположения из п. 2.1.3 ${ }^{\circ}$. Оставаясь на позициях формального анализа, возьмем множество

$$
\left\{x \in \Gamma_{\varepsilon}: s \in \mathscr{V}_{q}, s \geqslant s_{q}+d_{q} \Lambda^{-1}\right\}
$$

в качестве приближения повышенной точности к множеству $\left\{x \in \Xi^{+}(\varepsilon): s \in \mathscr{V}_{q}\right\}$. Вычислим величину $d_{q}$ в $(4.10)$.

Функции $\varphi_{i}$ и $\psi_{i}$ считаем гладкими. Вблизи $s_{q}$ имеем

$$
\varphi_{0}(s)-\psi_{0}(s)=b_{q}\left(s-s_{q}\right)+O\left(\left|s-s_{q}\right|^{2}\right) ;
$$


предположим, что $b_{q}=\partial_{s} \varphi_{q}\left(s_{q}\right)-\partial_{s} \psi_{0}\left(s_{q}\right)>0$. Найдем асимптотику функции $\gamma_{1}$ из (4.8) при $s \rightarrow s_{q}$. Могут возникнуть проблемы только со слагаемым $J \gamma_{0}$, однако благодаря равенству (2.10)

$$
J \gamma_{0}(s)=J \gamma_{0}\left(s_{q}\right)+O\left(\left|s-s_{q}\right|^{\beta}\right)
$$

при любом $\beta \in(0,1)$. Следовательно,

$$
\gamma_{1}(s)=B_{q}+O\left(\left|s-s_{q}\right|^{\beta}\right), \quad B_{q}=\pi\left(\varphi_{1}\left(s_{q}\right)-\psi_{1}\left(s_{q}\right)-J \gamma_{0}\left(s_{q}\right)\right) .
$$

Распространим определение $\gamma_{0}=\pi\left(\varphi_{0}-\psi_{0}\right)$ c $\left\{x \in \Gamma_{\varepsilon}^{+}: s \in \mathscr{V}_{q}\right\}$ на множество (4.10). Справедливо соотношение

$$
\pi\left(\varphi_{0}(s)+\psi_{0}(s)\right)+\Lambda^{-1} \gamma_{1}(s)=\pi b_{q}\left(s-s_{q}\right)+\Lambda^{-1} B_{q}+\cdots,
$$

где под многоточием подразумеваются младшие, несушественные члены. Сумма $\pi b_{q}\left(s-s_{q}\right)+\Lambda^{-1} B_{q}$ неотрицательна на множестве (4.10), если

$$
d_{q}=-\left(\pi b_{q}\right)^{-1} B_{q}
$$

Все формулы и заключения сохраняются и тогда, когда $b_{q}<0$. Случай $b_{q}=0$ не рассматривается.

Соотношения (4.10), (4.14) описывают формально главный член вариации множества $\Gamma_{\varepsilon}^{+}$вблизи точки $s_{q}$ (оно рассматривается как приближение к зоне контакта $\left.\Xi^{+}(\varepsilon)\right)$. Точно так же исследуются другие точки из $\Xi^{0}$. Обратим внимание на два обстоятельства. Во-первых, дополнительно требуется невырожденность коэффициента $b_{q}$ в разложении (4.11) около каждой из точек в $\Xi^{0}$. Во-вторых, из-за расширения (или сужения - это зависит от знака числа $\left.d_{q}(4.14)\right)$ области действия формулы $\gamma_{0}=\pi\left(\varphi_{0}-\psi_{0}\right)$ по сравнению с $(4.5)$ изменилось значение слагаемого $-\pi J \gamma_{0}$ в $(4.8)$, однако приращения длин дуг составляют $O\left(\Lambda^{-1}\right)$ и потому названным изменением можно пренебречь при определении $\gamma_{1}$. Именно благодаря последнему факту можно изучать каждую точку из $\Xi^{0}$ по отдельности, взаимное влияние вариаций множества $\Gamma_{\varepsilon}^{+}$не сказывается на первых двух членах асимптотики плотности.

4.3. Двучленная асимптотика решения результирующего неравенства (попытка вторая). Основываясь на формальном анализе, проведенном в предыдушем разделе, положим

$$
\widetilde{\gamma}(s)=\Lambda^{-1}\left(\pi\left(\varphi_{0}(s)-\psi_{0}(s)\right)+\Lambda^{-1} \gamma_{1}(s)\right)_{+},
$$

где $\gamma_{1}$ - величина (4.8), причем $\gamma_{0}$ определена формулой (4.5). Найдем невязку, определяемую плотностью (4.15) в вариационном неравенстве (4.6).

Если $\widetilde{\gamma}(s)>0$, то

$$
J \widetilde{\gamma}(s)-\pi^{-1} \widetilde{\gamma}(s) \ln (\varepsilon H(s))+\widetilde{Y}(s)=\varphi_{0}(s)-\psi_{0}(s)+\Lambda^{-1}\left(\varphi_{1}(s)-\psi_{1}(s)\right)
$$

(ср. с (2.29) и (4.7)); здесь

$$
\widetilde{Y}(s)=\pi^{-1}\left(\widetilde{\gamma}(s)-\Lambda^{-1} \gamma_{0}(s)\right) \ln H(s)+\Lambda^{-1} J \gamma_{0}(s)-J \widetilde{\gamma}(s)
$$


В случае $\widetilde{\gamma}(s)=0$, т.е. при $\pi\left(\varphi_{0}(s)-\psi_{0}(s)\right)+\Lambda^{-1} \gamma_{1}(s) \leqslant 0$, вьполнено неравенство

$$
J \widetilde{\gamma}(s)-\pi^{-1} \widetilde{\gamma}(s) \ln (\varepsilon H(s))+\widetilde{Y}(s) \geqslant \varphi_{0}(s)-\psi_{0}(s)+\Lambda^{-1}\left(\varphi_{1}(s)-\psi_{1}(s)\right)
$$

(cp. с (2.30) и (4.9)). Именно такие соотношения и требовались в (3.7) при определении понятия невязки.

Для использования теоремы 3.5 нужно оценить величину (3.8), т.е. норму функции $(4.17)$ в пространстве $W_{2}^{1}(\Gamma)$. Из-за присутствия в (4.17) оператора $J$, для которого неравенство

$$
\left\|J\left(\widetilde{\gamma}-\Lambda^{-1} \gamma_{0}\right) ; W_{2}^{1}(\Gamma)\right\| \leqslant c_{\tau}\left\|\widetilde{\gamma}-\Lambda^{-1} \gamma_{0} ; W_{2}^{1+\tau}(\Gamma)\right\|
$$

перестает выполняться в случае $\tau=0$, получить такую оценку авторам не удалось несмотря на явную малость разности $\widetilde{\gamma}-\Lambda^{-1} \gamma_{0}$. Даже само включение $\widetilde{\gamma} \in W_{2}^{1+\tau}(\Gamma)$ проблематично: операция $\sigma \mapsto(\sigma)_{+}$не является непрерывной на $W_{2}^{1+\tau}(\Gamma), \tau>0$.

4.4. Двучленная асимптотика решения вариационного неравенства (попытка третья, последняя и успешная). Предъявим дополнительные требования к данным задачи

$$
\varphi_{0} \in C^{2}(\Gamma), \quad \psi_{0} \in C^{2}(\Gamma) .
$$

Для того чтобы обеспечить включение (4.20) для следа $\psi_{0}$ на $\Gamma$ решения $V_{0}$ достаточно предположить, что правая часть $f_{0}$ принадлежит классу Гёльдера $C^{0, \varkappa}(\Omega)$ при каком-нибудь $\varkappa>0$, тогда $V_{0} \in C^{2, \varkappa}(\Omega \cap \mathscr{U}) \subset C^{2}(\Omega \cap \mathscr{U})$ (см. [31]). Пусть еще, как и ранее, вьполнены требования из п. $2.1 .3^{\circ}$, причем

$$
\partial_{s} \varphi_{0}(s)-\partial_{s} \psi_{0}(s) \neq 0 \quad \forall s \in \Xi^{0} .
$$

Для $f_{1}$ и $\varphi_{1}$ справедливы следующие условия: $f_{1} \in L_{p}(\Omega), p>3, \varphi_{1} \in C^{1}(\Gamma)$.

Воспользуемся конструкцией, вытекаюшей из формальных выкладок п. 4.2. Выберем разбиение единицы $\left\{\zeta_{0}, \zeta_{1}, \ldots, \zeta_{2 N}\right\}$ такое, что $\operatorname{supp} \zeta_{0} \cap \Xi^{0}=\varnothing$ и $\operatorname{supp} \zeta_{q}$ содержится в окрестности $\mathscr{V}_{q}$ точки $s_{q} \in \Xi^{0}$. Для определения асимптотического решения $\gamma_{*}$ нужно указать, как выплядят произведения $\zeta_{0} \gamma_{*}, \ldots, \zeta_{2 N} \gamma_{*}$.

Согласно сказанному в начале п. 4.2 при $q=0$ имеем

$$
\zeta_{0}(s) \gamma_{*}(s)= \begin{cases}\Lambda^{-1} \zeta_{0}(s) \gamma_{0}(s)+\Lambda^{-2} \zeta_{0}(s) \gamma_{1}(s), & s \in \Xi^{+} \\ 0, & s \in \Xi^{-}\end{cases}
$$

где $\gamma_{0}$ и $\gamma_{1}$ - функции (4.5) и (4.8). Следуя формальной схеме построения асимптотики зоны контакта, положим

$$
\begin{aligned}
& \zeta_{q}(s) \gamma_{*}(s)=\chi\left(\Lambda^{\alpha}\left(s-s_{q}\right)\right)\left(\Lambda^{-1} \pi\left(s-s_{q}\right) b_{q}+\Lambda^{-2} B_{q}\right)_{+} \\
& \quad+ \begin{cases}\left(1-\chi\left(\Lambda^{\alpha}\left(s-s_{q}\right)\right)\right)\left(\Lambda^{-1} \zeta_{q}(s) \gamma_{0}(s)+\Lambda^{-2} \zeta_{q}(s) \gamma_{1}(s)\right), & s \in \Xi^{+} \\
0, & s \in \Xi^{-}\end{cases}
\end{aligned}
$$

здесь $\chi$ - срезающая функция, введенная перед формулой $(3.2), \alpha$ - некоторое число из интервала $(0,1), b_{q}$ и $B_{q}$ - числа из представлений $(4.11)$ и (4.13). 
ЛЕмма 4.1. Если заменить $\widetilde{\gamma}$ на $\gamma_{*}$ и $\widetilde{Y}$ на $Y_{*}$, где

$$
\begin{aligned}
Y_{*}(s)= & \pi^{-1}\left(\gamma_{*}(s)-\Lambda^{-1} \gamma_{0}(s)\right) \ln H(s)-J \gamma_{*}(s)+\Lambda^{-1} J \gamma_{0}(s) \\
& +\sum_{q=1}^{2 N} \chi\left(\Lambda^{\alpha}\left(s-s_{q}\right)\right)\left(\varphi_{0}(s)-\psi(s)-\left(s-s_{q}\right) b_{q}+\Lambda^{-1} \pi^{-1}\left(\gamma_{1}(s)-B_{q}\right)\right)
\end{aligned}
$$

то соотночения (4.16) и (4.18) останутся верными, соответственно, при тех же условиях $\gamma_{*}(s)>0 u \gamma_{*}(s)=0$, что и прежде.

ДоКАЗАТЕЛЬСтво. Справедливость (4.16) проверяется прямой подстановкой формул (4.22)-(4.24) и (4.13). Более трудоемко установить (4.18), поскольку нужно рассмотреть несколько вариантов положения точки $s$.

Пусть сначала $s \in \mathscr{V}_{q}$ и $\chi_{q}(s) \equiv \chi\left(\Lambda^{\alpha}\left(s-s_{q}\right)\right)=1$. Тогда

$$
0=\gamma_{*}(s)=\pi\left(\Lambda^{-1}\left(s-s_{q}\right) b_{q}+\Lambda^{-2} \pi^{-1} B_{q}\right)_{+},
$$

т.е. $\left(s-s_{q}\right) b_{q}+\Lambda^{-1} \pi^{-1} B_{q} \leqslant 0$. После преобразований оказывается, что левая часть (4.18) равна

$$
\varphi_{0}(s)-\psi_{0}(s)+\Lambda^{-1}\left(\varphi_{1}(s)-\psi_{1}(s)\right)-\left(\left(s-s_{q}\right) b_{q}+\Lambda^{-1} \pi^{-1} B_{q}\right),
$$

видно, что она не менњше правой части.

Пусть теперь точка $s$ выбрана так, что $\chi_{q}(s)=0$ при всех $q$. Будемпользоваться вытекающими отсюда неравенствами $\left|s-s_{q}\right|>c \Lambda^{-\alpha}$ и требованием (4.21). Если $s \in \Xi^{+}$, то $\gamma_{0}(s)>C_{0} \Lambda^{-\alpha}$ и поэтому

$$
\gamma_{*}(s)=\Lambda^{-1} \gamma_{0}(s)+\Lambda^{-2} \gamma_{1}(s)>C_{0} \Lambda^{-1-\alpha}-C_{1} \Lambda^{-2} .
$$

Значит, включение $s \in \Xi^{+}$невозможно, т.е. $s \in \Xi^{-}$. Слева в (4.18) появляется выражение $\Lambda^{-1} J \gamma_{0}(s)$. Так как

$$
\varphi_{0}(s)-\psi_{0}(s)<-C_{0} \Lambda^{-\alpha}
$$

и $\alpha \in(0,1)$, то при малом $\varepsilon$ (большом $\Lambda$ ) соотношение (4.18) выполнено.

Пусть, наконец, $s \in \mathscr{V}_{q}$ и $0<\chi_{q}(s)<1$. По-прежнему, $\left|s-s_{q}\right|>c \Lambda^{-\alpha}$. Функция $\gamma_{*}$ определена формулой (4.23). Таким образом, $s \in \Xi^{-}$и

$$
\left(s-s_{q}\right) b_{q}+\Lambda^{-1} \pi^{-1} B_{q} \leqslant 0 .
$$

Применяя (4.8) и (4.24), заключаем, что (4.18) эквивалентно следуюшему неравенству

$$
\begin{aligned}
\left(1-\chi_{q}(s)\right) \Lambda^{-1} J \gamma_{0}(s) & -\chi_{q}(s)\left(\left(s-s_{q}\right) b_{q}+\Lambda^{-1} \pi^{-1} B_{q}\right) \\
& \geqslant\left(1-\chi_{q}(s)\right)\left(\varphi_{0}(s)-\psi_{0}(s)+\Lambda^{-1}\left(\varphi_{1}(s)-\psi_{1}(s)\right)\right) .
\end{aligned}
$$

Осталось заметить, что последняя формула верна благодаря (4.25) и (4.26).

Итак, $\gamma_{*}$ - асимптотическое решение результируюшего вариационного неравенства (4.6), причем его невязка имеет вид (4.24). Необходимо оценить величину $\delta_{Y}$, 
т.е. норму $Y_{*}$ в пространстве $W_{2}^{1}(\Gamma)$. Так как $J: W_{2}^{1+\varkappa}(\Gamma) \rightarrow W_{2}^{1}(\Gamma)-$ непрерывное отображение при любом $\varkappa>0$, то достаточно изучить выражения

$$
\delta_{*}=\left\|\gamma_{*}-\Lambda^{-1} \gamma_{0} ; W_{2}^{1+\varkappa}(\Gamma)\right\|, \quad \delta_{q}=\left\|Z_{q} ; W_{2}^{1}(\Gamma)\right\|,
$$

где $Z_{q}$ - члены суммы по $q=1, \ldots, 2 N$ из (4.24).

Подчеркнем, что $\gamma_{0} \in W_{2}^{1+\tau}(\Gamma)$ при $\tau \in\left(0, \frac{1}{2}\right)$ (производная $\partial_{s} \gamma_{0}-$ кусочно-гладкая функция с конечным набором точек разрьва первого рода). Согласно (4.8) то же самое верно и для $\gamma_{1}$ (оператор $J: W_{2}^{1+\tau_{1}}(\Gamma) \rightarrow W_{2}^{1+\tau_{2}}(\Gamma)$, $0<\tau_{1}<\tau_{2}<\frac{1}{2}$, непрерьвен). Рассмотрим вторую норму в (4.27). Ввиду условия (4.20)

$$
\left|\varphi_{0}(s)-\psi_{0}(s)-\left(s-s_{q}\right) b_{q}\right| \leqslant c\left|s-s_{q}\right|^{2}, \quad\left|\partial_{s} \varphi_{0}(s)-\partial_{s} \psi_{0}(s)-b_{q}\right| \leqslant c\left|s-s_{q}\right| .
$$

Следовательно, оценивая подынтегральные выражения по максимуму модуля, находим

$$
\left\|\chi_{q}\left(\varphi_{0}-\psi_{0}-\left(s-s_{q}\right) b_{q}\right) ; W_{2}^{1}(\Gamma)\right\| \leqslant c \Lambda^{-3 \alpha / 2} .
$$

В силу (4.13)

$$
\left\|\left(\varphi_{1}-\psi_{1}-B_{q}\right) \partial_{s} \chi_{q} ; L_{2}(\Gamma)\right\| \leqslant c \Lambda^{-\alpha(\beta-1 / 2)},
$$

где $\beta \in(0,1)$. Принимая во внимание одномерное дробное неравенство Харди

$$
\left\|\partial_{s} \gamma_{1} ; W_{2}^{\tau}(\Gamma)\right\| \geqslant c\left\|\left|s-s_{q}\right|^{-\tau} \partial_{s} \gamma_{1} ; L_{2}(\Gamma)\right\|
$$

(см. [32]), выводим

$$
\begin{aligned}
\left\|\chi_{q} \partial_{s} \gamma_{1} ; L_{2}(\Gamma)\right\| & \leqslant\left\|\partial_{s} \gamma_{1} ; L_{2}\left(\operatorname{supp} \chi_{q}\right)\right\| \\
& \leqslant c \Lambda^{-\tau \alpha}\left\|\left(s-s_{q}\right)^{-\tau} \partial_{s} \gamma_{1} ; L_{2}\left(\operatorname{supp} \chi_{q}\right)\right\| \\
& \leqslant c \Lambda^{-\tau \alpha}\left\|\gamma_{1} ; W_{2}^{1+\tau}(\Gamma)\right\| .
\end{aligned}
$$

Формулы (4.29) и (4.30) вместе с одномерным неравенством Фридрихса (произведение $\chi_{q}\left(\varphi_{1}-\psi_{1}-B_{q}\right)$ имеет малый носитель) дают оценку для нормы $\left\|\chi_{q}\left(\varphi_{1}-\psi_{1}-B_{q}\right) ; W_{2}^{1}(\Gamma)\right\|$. Считая $\beta=\tau+1 / 2 \in(0,1)$ в (4.29) и используя (4.28), получаем окончательно, что

$$
\left\|Z_{q} ; W_{2}^{1}(\Gamma)\right\| \leqslant c\left(\Lambda^{-3 \alpha / 2}+\Lambda^{-1-\tau \alpha}\right) .
$$

Теперь рассмотрим первую величину в (4.27), т.е. оценим нормы функций

$$
z_{q}=\zeta_{q} \gamma_{*}-\Lambda^{-1} \zeta_{q} \gamma_{0}, \quad q=0,1, \ldots, 2 N
$$

в пространстве $W_{2}^{1+\varkappa}(\Gamma)$. В силу формулы $(4.22)$ и сказанного ранее о $\gamma_{1}$,

$$
\left\|z_{0} ; W_{2}^{1+\varkappa}(\Gamma)\right\|=\Lambda^{-2}\left\|\zeta_{0} \gamma_{1} ; W_{2}^{1+\varkappa}(\Gamma)\right\| \leqslant c \Lambda^{-2} .
$$

Пусть $q=1, \ldots, 2 N$. Согласно (4.23) и (4.5) производная $\partial_{s} z_{q}$ равна нулю вне объединения множеств $(4.10)$ и $\Gamma_{\varepsilon}^{+}$, а на нем есть сумма следующих слагаемых:

$$
\begin{aligned}
& I_{1}(s)=\left(1-\chi_{q}(s)\right) \Lambda^{-2} \partial_{s}\left(\zeta_{q}(s) \gamma_{1}(s)\right), \\
& I_{2}(s)=\chi_{q}(s) \Lambda^{-1}\left(\pi b_{q} \Theta\left(s-s_{q}\right)-\partial_{s} \gamma_{0}(s)\right), \\
& I_{3}(s)=\Lambda^{-1} \pi b_{q}\left(\Theta\left(s-s_{q}-\Lambda^{-1} d_{q}\right)-\Theta\left(s-s_{q}\right)\right), \\
& I_{4}(s)=\partial_{s} \chi_{q}(s)\left(\Lambda^{-1} \pi\left(\left(s-s_{q}\right) b_{q}-\varphi_{0}(s)-\psi_{0}(s)\right)+\Lambda^{-2}\left(B_{q}-\gamma_{1}(s)\right)\right),
\end{aligned}
$$


здесь $\Theta$ - функция Хевисайда, а $d_{q}$ - число (4.14). Отметим, что $\partial_{t}(t)_{+}=\Theta(t)$, а множитель $\chi_{q}(s)$ в выражении для $I_{3}$ опушен, так как он равен единице на $\operatorname{supp} I_{3}$.

Проще всего оценить норму $I_{4}$. Аналогично (4.28) и (4.30) имеем

$$
\left\|I_{4} ; W_{2}^{\varkappa}(\Gamma)\right\| \leqslant c\left\|I_{1} ; W_{2}^{1}(\Gamma)\right\| \leqslant c \Lambda^{\alpha}\left(\Lambda^{-1-3 \alpha / 2}+\Lambda^{-2-\tau \alpha}\right) .
$$

Нормы в $L_{2}(\Gamma)$ слагаемых $I_{1}, I_{2}$ и $I_{3}$ суть $O\left(\Lambda^{-2}\right), O\left(\Lambda^{-1-3 \alpha / 2}\right)$ и $O\left(\Lambda^{-3 / 2}\right)$ : в первом случае соответствуюшее неравенство очевидно, во втором-нужно учесть, что длина supp $I_{2}$ есть $O\left(\Lambda^{-\alpha}\right)$ и что ввиду $(4.5),(4.11)$ и (4.20)

$$
\left|\pi b_{q} \Theta\left(s-s_{q}\right)-\partial_{s} \gamma_{0}(s)\right| \leqslant c\left|s-s_{q}\right|,
$$

а в третьем - достаточно заметить, что $I_{3}$ - “ступенька" длиной $O\left(\Lambda^{-\alpha}\right)$ и высотой $O\left(\Lambda^{-1}\right)$. Таким образом, осталось оценить полунормы

$$
\left\|I_{i}\right\| \mid=\left(\int_{\Gamma} \int_{\Gamma}|s-t|^{-1-2 \varkappa}\left|I_{i}(s)-I_{i}(t)\right|^{2} d s d t\right)^{1 / 2} .
$$

Пользуясь тем, что $\left|\chi_{q}(s)-\chi_{q}(t)\right| \leqslant c \Lambda^{\alpha \nu}|s-t|^{\nu}$ для $\nu=2 \varkappa \in(0,1)$ при $i=1$, имеем

$$
\begin{aligned}
\left.\left\|I_{1}\right\|\right|^{2} & \leqslant \Lambda^{-4} \int_{\Gamma} \int_{\Gamma}|s-t|^{-1-2 \varkappa}\left|\chi_{q}(s)-\chi_{q}(t)\right|^{2}\left|\partial_{s}\left(\zeta_{q}(s) \gamma_{1}(s)\right)\right|^{2} d s d t \\
& +\Lambda^{-4} \int_{\Gamma} \int_{\Gamma}|s-t|^{-1-2 \varkappa}\left|1-\chi_{q}(t)\right|^{2}\left|\partial_{s}\left(\zeta_{q}(s) \gamma_{1}(s)\right)-\partial_{t}\left(\zeta_{q}(t) \gamma_{1}(t)\right)\right|^{2} d s d t \\
& \leqslant c \Lambda^{-4}\left(\Lambda^{4 \alpha \varkappa}\left\|\partial_{t} \zeta_{q} \gamma_{1} ; L_{2}(\Gamma)\right\|^{2}+\left\|\partial_{s} \zeta_{q} \gamma_{1} ; W_{2}^{\varkappa}(\Gamma)\right\|\right) \leqslant C \Lambda^{4 \alpha \varkappa-4} .
\end{aligned}
$$

Для функции $s \mapsto F(s)=\pi b_{q} \Theta\left(s-s_{q}\right)-\partial_{s} \gamma_{0}(s)$ кроме (4.33) верно неравенство $|F(s)-F(t)| \leqslant C|s-t|$. Поэтому в случае $i=2$

$$
\begin{aligned}
\left.\left\|I_{2}\right\|\right|^{2} \leqslant & \Lambda^{-2} \int_{\Gamma} \int_{\Gamma}|s-t|^{-1-2 \varkappa}\left|\chi_{q}(s)-\chi_{q}(t)\right|^{2}|F(s)|^{2} d s d t \\
& +\Lambda^{-2} \int_{\Gamma} \int_{\Gamma}|s-t|^{-1-2 \varkappa}\left|\chi_{q}(t)\right|^{2}|F(s)-F(t)|^{2} d s d t \\
\leqslant & c \Lambda^{-2}\left(\Lambda^{4 \alpha \varkappa} \int_{L_{q}} \int_{\Gamma}|s-t|^{-1+2 \varkappa}|t|^{2} d s d t\right. \\
& \left.+\Lambda^{4 \alpha \varkappa} \int_{\Gamma} \int_{L_{q}}|s-t|^{-1+2 \varkappa}|t|^{2} d s d t+\int_{L_{q}} \int_{\Gamma}|s-t|^{1-2 \varkappa} d s d t\right) \\
\leqslant & c \Lambda^{-2}\left(\Lambda^{\alpha(4 \varkappa-1)}+\Lambda^{\alpha(4 \varkappa-3)}+\Lambda^{-\alpha}\right) \leqslant c \Lambda^{-2-\alpha(1-4 \varkappa)},
\end{aligned}
$$

здесь $L_{q}=\operatorname{supp} \chi_{q}=\left(s_{q}-c \Lambda^{-\alpha}, s_{q}+c \Lambda^{-\alpha}\right)$.

Наконец, выражение $\left\|I_{3}\right\|$ вычисляется непосредственно. Пусть, например, $d_{q}>0$. Положим $\Delta=\left(s_{q}, s_{q}+\Lambda^{-1} d_{q}\right), \bar{\Delta}=\operatorname{supp} I_{3}$. Подынтегральное выражение в (4.34), $i=3$, является кусочно-постоянной функцией, носителем которой служит объединение множеств $\bar{\Delta} \times(\Gamma \backslash \Delta)$ и $(\Gamma \backslash \Delta) \times \bar{\Delta}$. Сводя двойной интеграл к повторному, после несложного интегрирования, получаем

$$
\left\|I_{3}\right\| \leqslant c \Lambda^{-2}\left|d_{q} \Lambda^{-1}\right|^{1-2 \varkappa}=C \Lambda^{-3+2 \varkappa} .
$$

Итак, собирая все проверенные соотношения для $I_{m}$ и ссылаясь на неравенство Фридрихса при рассмотрении $\left\|z_{q} ; L_{2}(\Gamma)\right\|$, приходим к формулам

$$
\begin{aligned}
\left\|z_{q} ; W_{2}^{1+\varkappa}(\Gamma)\right\| \leqslant c\left(\Lambda^{-1-\alpha / 2}+\Lambda^{-2-\alpha(1-\tau)}+\Lambda^{-2+2 \alpha \varkappa}\right. \\
\left.+\Lambda^{-1+\alpha(2 \varkappa-1 / 2)}+\Lambda^{2 \varkappa-3 / 2}\right), \quad q=1, \ldots, 2 N,
\end{aligned}
$$

которые и требовались для оценки $\delta_{*}$ в $(4.27)$. 
4.5. Обоснование двучленной асимптотики. Нетрудно увидеть, что при любом $\rho>0$ можно выбрать числа $\alpha, \tau$ и $\varkappa$ столь близко к $1-0,1 / 2-0$ и +0 , соответственно, что правые части неравенств (4.31) и (3.35) можно заменить на $c \Lambda^{\rho-3 / 2}$. Из этих неравенств и из (4.32) вытекает, что величины (4.27) имеют мажоранты того же вида. Таким образом,

$$
\delta_{Y}=\left\|Y_{*} ; W_{2}^{1}(\Gamma)\right\| \leqslant c \Lambda^{\rho-3 / 2} .
$$

Заметим, что $\delta_{o}=O(1)$ согласно (3.17) и (4.1). Наконец, полученная оценка для $\left\|\gamma_{*}-\Lambda^{-1} \gamma_{0} ; W_{2}^{1+\tau}(\Gamma)\right\|$ (нет ограничений на $\varkappa=\tau$ ) и очевидная формула $\left\|\Lambda^{-1} \gamma_{0} ; W_{2}^{1+\tau}(\Gamma)\right\|=O\left(\Lambda^{-1}\right)$ приводят к соотношению $\delta_{\gamma}=O\left(\Lambda^{-1}\right)$ (см. (3.18)).

Теперь можно применить теорему 3.5 и сформулировать следуюшее утверждение.

ТЕОРема 4.2. Пусть выполнены сформулированные в $n .2 .1 .3^{\circ}$ ограничения, а также соотношения (4.1), (4.2), (4.20), (4.21). Пусть еще $U_{*}-$ глобальное асимптотическое приближение (3.3) к решению и задачи (1.2)-(1.5), причем плотность $\gamma_{*}$ определена равенствами (4.22) и (4.23), $v_{*}$ - решение (2.8) задачи (2.1) при $\gamma=\gamma_{*} u V=V_{0}+\Lambda^{-1} V_{1}, V_{i}$ - ограниченные решения задачи (2.1) с правыми частями $f_{i}$ из (4.1), $E$ - функиия (2.22), $a_{*}$ - величина (3.9) при $Y=Y_{*}$ (см. (4.24)), а $X_{\varepsilon}$ и $\chi_{\varepsilon}-$ срезающие функции (3.2). Тогда при любом $\rho>0$ справедливо неравенство

$$
\left\|\nabla_{x} u-\nabla_{x} U_{*} ; L_{2}(\Omega)\right\| \leqslant c_{\rho}|\ln \varepsilon|^{\rho-3 / 2},
$$

в котором постоянная $c_{\rho}$ не зависит от $\varepsilon \in\left(0, \varepsilon_{0}\right), \varepsilon_{0}>0$.

Рассуждения, подобные тем, которые сопутствовали формулам (3.33), позволяют заключить, что оценка (4.36) оправдывает присутствие в глобальном асимптотическом приближении слагаемых $V_{0}, \Lambda^{-1} V_{1}$ и сингулярного решения, порожденного плотностью $\gamma_{*}$ из $(4.22),(4.23)$. То же касается и главного члена пограничного слоя, который уже был построен в $\S 2$, однако поправка, определяемая найденной в данном параграфе разностью $\gamma_{*}-\Lambda^{-1} \gamma_{0}$, выходит за пределы точности, гарантируемой теоремой 4.2. Тем не менее, построение двучленной асимптотики плотности $\gamma_{*}$ обязательно - без нее нельзя указать сингулярное решение $v_{*}$. Вероятно, потеря неравенством (4.36) предполагавшейся точности происходит вследствие возникновения трехмерного пограничного слоя, сконцентрированного вблизи множества $\Xi^{0} \subset \Gamma$, однако никаких конкретных результатов в этом направлении нет.

\section{§5. Теоремы о сходимости}

5.1. Ограниченное решение $V(x)$ как предел решения $u(\varepsilon, x)$. Пусть вьполнены условия теоремы 3.6. Заметим, что согласно сказанному в начале п. 2.1

$$
\left\|\chi_{\varepsilon}(V-\psi) ; W_{2}^{1}(\Omega)\right\|=o(1), \quad \varepsilon \rightarrow 0 .
$$

Преобразуем формулу (3.3) для $U(\varepsilon, x)$ к виду

$$
\begin{aligned}
U=V & +\chi_{\varepsilon}(V-\psi)+X_{\varepsilon}\left(v_{*}-V+\chi \gamma_{*} \pi^{-1} \ln r\right) \\
& +\chi X_{\varepsilon} \gamma_{*} \pi^{-1}(\ln (\varepsilon+r)-\ln r) \\
& +\chi \gamma_{*} \pi^{-1} \ln (\varepsilon+r)+\chi_{\varepsilon} \gamma_{*}\left(\pi^{-1} \ln (1+\rho)-E\right)
\end{aligned}
$$


(воспользовались (3.4), (3.2), а под $\chi$ понимаем не зависящую от $\varepsilon$ срезаюшую функцию $x \mapsto \chi(r)$ с носителем в $\mathscr{U} ; \chi(r)=1$ вблизи $r=0)$. Так как $\gamma_{*}(s)=O\left(\Lambda^{-1}\right)($ см. $(3.1))$, то в силу равенства $(2.8)$ и предложения 2.1

$$
\left\|X_{\varepsilon}\left(v_{*}-V+\gamma_{*} \pi^{-1} \ln r\right) ; W_{2}^{1}(\Omega)\right\|=o(1), \quad \varepsilon \rightarrow 0 .
$$

Рассмотрим оставшиеся слагаемые $\Sigma_{4}, \Sigma_{5}$ и $\Sigma_{6}$ справа в (5.2). Непосредственные вычисления с учетом (3.1) приводят к соотношениям

$$
\left\|\Sigma_{p} ; W_{2}^{1}(\Omega)\right\|=o(1), \quad \varepsilon \rightarrow 0,
$$

для $p=4$ и $p=5$. Обрашаясь к $(2.22)-(2.25)$, видим, что $\left|E(\eta ; s)-\pi^{-1} \ln (1+\rho)\right| \leqslant c$,

$$
\begin{aligned}
& \left|\nabla_{\eta} E(\eta ; s)-\pi^{-1} \nabla_{\eta} \ln (1+\rho)\right| \\
& \quad \leqslant c(1+\rho)^{-1} \max \left\{1,\left(\left(\eta_{1}+h_{-}(s)\right)^{2}+\eta_{2}^{2}\right)^{-1 / 4},\left(\left(\eta_{1}-h_{+}(s)\right)^{2}+\eta_{2}^{2}\right)^{-1 / 4}\right\} .
\end{aligned}
$$

Таким образом, повторяя рассуждения, предшествующие (3.20), получаем соотношение (5.4) и в случае $p=6$. Следовательно, в силу (5.1)-(5.4) функции $U(\varepsilon, \cdot)$ сходятся по норме $W_{2}^{1}(\Omega)$ при $\varepsilon \rightarrow+0$ к решению $V \in W_{2}^{1}(\Omega)$ задачи $(2.1)$. Вместе с теоремой 3.6 этот факт доказывает следуюшую теорему о сходимости.

Теорема 5.1. В предположениях из $n .2 .1$ и n. 3.1 решение $и(\varepsilon, \cdot)$ задачи (1.2)-(1.5) и ограниченное решение $V$ задачи (2.1) связаны соотночением

$$
\left\|u(\varepsilon, \cdot)_{-} V ; W_{2}^{1}(\Omega)\right\| \rightarrow 0 \quad n p u \quad \varepsilon \rightarrow+0,
$$

т.е. первое стремится ко второму в энергетической метрике.

Итак, в ситуации из $\S 2$ вариационное неравенство (1.7) "не замечает" в пределе препятствия на $\Gamma_{\varepsilon}$ и его решение "преврашается" в решение линейной задачи. Это согласуется с результатами [33], поскольку емкость $\Gamma_{\varepsilon}$ исчезает при $\varepsilon \rightarrow+0$.

5.2. Сингулярное решение $v(x)$ как предел решения $u(\varepsilon, x)$. Попытаемся так изменить данные задачи (1.2)-(1.5), чтобы пределом $u(\varepsilon, \cdot)$ служило решение $v$ задачи (2.1) с $\delta$-функцией, распределенной вдоль $\Gamma$. Функция $v$ принадлежит $L_{2}(\Omega)$, но не попадает в $W_{2}^{1}(\Omega)$. Поэтому рассчитывать на сходимость в пространстве $W_{2}^{1}(\Omega)$ не приходится.

Пусть

$$
f(\varepsilon, x)=F(x), \quad \varphi(\varepsilon, s)=\Lambda \Phi(s) .
$$

Так как неравенство (1.7) допускает умножение на положительную постоянную (т.е. $\Lambda^{-1} u$ - решение задачи с данными $\Lambda^{-1} f$ и $\Lambda^{-1} \varphi$ ), то условия (5.5) можно привести к виду (4.1), при этом

$$
f_{1}=F, \quad f_{0}=\widetilde{f}=0, \quad \varphi_{0}=\Phi, \quad \varphi_{1}=\widetilde{\varphi}=0 .
$$

Предположим, что $F \in L_{p}(\Omega), p>3$, и $\Phi \in C^{2}(\Gamma)$. Поскольку $V_{0}=0$ и $\psi_{0}=0$, условие (4.20) вьполнено. Пусть еще функция $\Phi$ имеет нули в конечном числе точек, и в каждой из них производная $\partial_{s} \Phi$ не вырождается (выполнены требования из п. 2.1.3 ${ }^{\circ}$ и неравенства (4.21)). Теперь можно воспользоваться результатами $\S 4$ : построить плотность $\gamma_{*}$, сингулярное решение $v_{*}$ и глобальное приближение $U_{*}$ так, чтобы

$$
\left\|\Lambda^{-1} u-U_{*} ; W_{2}^{1}(\Omega)\right\|=o(1) \text { при } \varepsilon \rightarrow+0 .
$$


Пусть $\gamma_{0}=\pi(\Phi)_{+}, V_{1} \in W_{2}^{1}(\Omega)$ - решение задачи $(2.1)$ с правой частью $F$ и

$$
v(x)=V_{1}(x)+\pi \int_{\Gamma} G(x ; s)(\Phi(s))_{+} d s .
$$

Заметим, что $G(\cdot ; s) \in L_{2}(\Omega)($ см. $(2.7))$ и зависимость величины $\left\|G(\cdot ; s) ; L_{2}(\Omega)\right\|$ от $s$ непрерывная. Кроме того, в п. 4.4 при рассмотрении $\delta_{*}$ из (4.26) было установлено, что $\left\|\gamma_{*}-\Lambda^{-1} \gamma_{0} ; L_{2}(\Gamma)\right\|=o\left(\Lambda^{-1}\right)$. Таким образом,

$$
\left\|v_{*}-\Lambda^{-1} v ; L_{2}(\Omega)\right\|=o\left(\Lambda^{-1}\right) .
$$

Наконец, те же аргументы, что и в п. 5.1, позволяют получить соотношение

$$
\left\|U_{*}-v_{*} ; L_{2}(\Omega)\right\|=o\left(\Lambda^{-1}\right) .
$$

Используя (5.6), (5.8), (5.9), заключаем, что

$$
\left\|u-v ; L_{2}(\Omega)\right\|=o(1), \quad \varepsilon \rightarrow+0 .
$$

ТЕОРема 5.2. При сделанных выше предположсениях решение $и(\varepsilon, \cdot)$ задачи (1.2)-(1.5) и решение (5.7) задачи (2.1) связаны формулой (5.10), т.е. первое сходится ко второму в метрике пространства $L_{2}(\Omega)$.

Вероятнее всего, утверждение теоремы 5.2 верно и при более слабых ограничениях на функции $F$ и $\Phi$ из (5.5), однако наши возможности ограничены априорной оценкой решения вариационного неравенства (см. п. 3.4 и, в частности, леммы 3.2 и 3.3). Недостаток используемой оценки в том, что она не учитывает зону контакта, на которой реализовались условия Дирихле, но пути ее улучшения не прослеживаются. По тем же причинам не удается обосновать асимптотическую конструкцию, предлагаемую в следуюшем параграфе.

\section{§6. Формальная асимптотика решения задачи Дирихле в случае отсутствия условий Дирихле $(\Sigma=\varnothing)$}

6.1. Обсуждение проблемы. Известно (см.,например, [2]), что решение задачи Синьорини (1.2), (1.4) (1.5), в которой $\Sigma=\varnothing$, сушествует не всегда. Необходимое условие существования $(f, 1)_{\Omega} \leqslant 0$ легко выводится из формулы Грина. Мы введем более сильное ограничение на правую часть уравнения (1.2)

$$
(f, 1)_{\Omega}<0,
$$

обеспечиваюшее вдобавок и единственность решения (см. [2]).

Наиболее интересной оказывается ситуация, в которой зависимость от $\varepsilon$ конкретизируется следующим образом

$$
\varphi(\varepsilon, s)=\Lambda \varphi_{0}(s), \quad \Lambda=|\ln \varepsilon|,
$$

здесь $\varphi_{0} \in C^{1}(\Gamma)$. Пусть, к тому же, $f \in L_{p}(\Omega), p>3$, не зависит от $\varepsilon$.

Условие $\Sigma=\varnothing$ несколько усложняет (по сравнению с $\S 2$ ) алгоритм построения асимптотики. К примеру, первая предельная задача (2.1) оказывается задачей Неймана и уже не имеет решения в классе $W_{2}^{1}(\Omega)$ ввиду предположения (6.1). Это препятствие преодолимо, если, как и ранее, в качестве решений допустить функции с логарифмическими особенностями на $\Gamma$, а плотность $\gamma$ из (2.6) подчинить равенству

$$
(\gamma, 1)_{\Gamma}=-(f, 1)_{\Omega}
$$

гарантируюшему возможность построить решение (2.6) задачи (2.1). Все остальные элементы асимптотических конструкций такие же, как и в $\S 2$. Посему избираем конспективный стиль изложения и будем в основном фиксировать отличия. 
6.2. Главный член внешнего разложения. Под $G(\cdot ; s)$ подразумеваем обобщенную функцию Грина с особенностью в точке $\mathbf{s} \in \Gamma$ с координатой $s$, удовлетворяюшую (см. [34]) соотношениям

$$
\begin{gathered}
\Delta_{x} G(x ; s)=\left(\operatorname{mes}_{3} \Omega\right)^{-1}, \quad x \in \Omega ; \\
\partial_{n} G(x ; s)=0, \quad x \in \partial \Omega \backslash \mathbf{s} ; \\
G(x ; s)=(2 \pi|x-\mathbf{s}|)^{-1}+o\left(|x-\mathbf{s}|^{\delta-1}\right), \quad x \rightarrow \mathbf{s}, \quad \delta \in(0,1) ; \\
(G(\cdot ; s), 1)_{\Omega}=0 .
\end{gathered}
$$

Пусть $v_{0}$ - ограниченное решение задачи (2.1) с новой правой частью $f-\left(\operatorname{mes}_{3} \Omega\right)^{-1}(f, 1)_{\Omega}$, причем $\left(v_{0}, 1\right)_{\Omega}=0$.

Формулу (2.8) для главного члена внешнего разложения заменим следующей

$$
v(x)=A|\ln \varepsilon|+v_{0}(x)+\int_{\Gamma} \gamma(t) G(x ; t) d t,
$$

здесь $A$-постоянная, подлежащая определению. Так как константа удовлетворяет однородным уравнениям (2.1) (напоминаем еще раз, что $\Sigma=\varnothing$ и условия Дирихле отсутствуют), то сумма (6.4) оказывается решением задачи $(2.1),(2.6)$, благодаря (6.3) члены, содержащие теs 3 , взаимно сокрашаются.

Справедливо аналогичное (2.9) асимптотическое представление

$$
v(x)=-\pi^{-1} \gamma(s) \ln r+J \gamma(s)+A|\ln \varepsilon|+v_{0}(s)+O(r|\ln r|), \quad r \rightarrow 0,
$$

а оператор $J$ строится по обобщенной функции Грина в соответствии с (2.10).

6.3. Плоский пограничный слой. Процедура вывода второй предельной задачи (2.18)-(2.20) и ее исследование не претерпевают каких-либо изменений, однако итоговые формулы модифицируются. Так, согласно (6.5) вместо (2.21) возникает асимптотическое условие

$$
e(\eta ; s)=-\pi^{-1} \gamma(s) \ln (\varepsilon \rho)+J \gamma(s)+A|\ln \varepsilon|+v_{0}(s)+o(1), \quad \rho \rightarrow \infty,
$$

и, соответственно, равенства $(2.27),(2.28)$ заменяются следуюшим

$$
e(\eta ; s)=-\gamma(s) E(\eta ; s)-\pi^{-1} \gamma(s) \ln (\varepsilon H(s))+J \gamma(s)+A|\ln \varepsilon|+v_{0}(s) .
$$

Наконец, сравнивая (6.6) и (6.2) с учетом (2.20), приходим к совокупности соотношений

$$
\begin{gathered}
\gamma(s) \geqslant 0, \\
\gamma(s)>0 \Longrightarrow J \gamma(s)+v_{0}(s)-\pi^{-1} \gamma(s) \ln (\varepsilon H(s))=\Lambda\left(\varphi_{0}(s)-A\right), \\
\gamma(s)=0 \Longrightarrow J \gamma(s)+v_{0}(s)-\pi^{-1} \gamma(s) \ln (\varepsilon H(s)) \geqslant \Lambda\left(\varphi_{0}(s)-A\right),
\end{gathered}
$$

которые вместе с (6.3) формируют результирующую задачу для определения неизвестных плотности $\gamma$ и постоянной $A$ в (6.4). 
6.4. Асимптотическое решение результируюшей задачи. Соотношения (6.7) и (6.3), разумеется, можно переписать как вариационное неравенство, однако мы ограничимся указанием главного (относительно $\Lambda$ ) члена $\left\{\gamma_{*}, A_{*}\right\}$ асимптотического решения.

Поступая так же, как в начале п. 3.1, положим

$$
\gamma_{*}=\pi\left(\varphi_{0}-A_{*}\right)_{+} \cdot
$$

Осталось обеспечить условие (6.3), которое согласно (6.8) принимает вид

$$
\pi \int_{\Gamma}\left(\varphi_{0}(s)-A_{*}\right)_{+} d s=-\int_{\Omega} f(x) d x
$$

и служит для вычисления $A_{*}$.

ПРЕДЛОЖЕНИЕ 6.1. При выполнении (6.1) уравнение (6.9) имеет единственное решение $A_{*}$.

ДокАЗАТЕЛЬСТво. Учитывая включение $\varphi_{0} \in C^{1}(\Gamma)$, находим

$$
\frac{d}{d A} \int_{\Gamma}\left(\varphi_{0}(s)-A\right)_{+} d s=-\operatorname{mes}_{1}\left\{s \in \Gamma: \varphi_{0}(s) \geqslant A\right\} .
$$

Таким образом, непрерывная функция

$$
\left(-\infty, \max \left\{\varphi_{0}(s): s \in \Gamma\right\}\right) \ni A \mapsto \int_{\Gamma}\left(\varphi_{0}(s)-A\right)_{+} d s
$$

строго монотонно убывает, а ее область значений совпадает с $(0,+\infty)$. Этих фактов достаточно для нужного вывода.

Итак, определяя $A_{*}$ и $\gamma_{*}$ из (6.9) и (6.8), по формулам (6.4) и (6.6) восстанавливаем главные члены $v_{*}$ и $e_{*}$ внешнего и внутреннего разложений.

\section{Список литературы}

1. Лионс Ж.-Л. Некоторые методы решения нелинейных краевых задач. М.: Мир, 1972.

2. Дюво Г., Лионс ЖК.-Л. Неравенства в механике и физике. М.: Наука, 1980.

3. Гловински $P$., Лионс ЖК.-Л., Тремольер $Р$. Численное исследование вариационных неравенств. М.: Мир, 1979.

4. Назаров С.А. Асимптотическое решение вариационных неравенств для линейного оператора с мальм параметром при старшшх производных // Изв. АН СССР. Сер. матем. 1980. T. 54. № 4. C. $754-773$.

5. Назаров С. А. Асимптотическое решение вариационного неравенства, моделируюшего трение // Изв. АН СССР. Сер. матем. 1990. Т. 54. № 5. С. 990-1020.

6. Назаров С. А. О возмущении решений задачи Синьорини // Матем. заметки. 1990. T. 47. №1. C. 115-126.

7. Назаров C. А. Асимптотика решений задачи Синьорини без трения или смальмм трением // Проблемы матем. анализа. Вьп. 12. СПб.: Изд-во СПбГУ, 1992. С. 82-109.

8. Назаров C.A. Эллиптические неравенства для тонких упругих областей при малом коэффициенте трения // Диффференц. уравнения. 1990. Т. 26. №4. С. 667-687.

9. Аргатов И. И., Назаров С. А. Асимптотическое решение задачи Синьорини с мальми участками свободной границы // Сиб. матем. журн. 1994. Т. 35. № 2. С. 258-277.

10. Аргатов И.И., Назаров С.А. Асимптотическое решение задачи об упругом теле, лежащем на нескольких малых опорах // Прикладная матем. и мех. 1994. Т. 58. № 2 . C. $110-118$. 
11. Федорюк M. В. Задача Дирихле для оператора Лапласа во внешности тонкого тела вращения // Труды сем. им. С. Л. Соболева. Новосибирск: Институт математики СО АН CCCP, 1980. № 1. C. 113-131.

12. Федорюк M. В. Асимптотика решения задачи Дирихле для уравнений Лапласа и Гельмгольца во внешности тонкого цилиндра // Изв. АН СССР. Сер. матем. 1981. Т. 45. № 1. C. $167-186$.

13. Мазья В.Г., Назаров С.А., Пламеневский Б. А. Об асимптотике решений задачи Дирихле в трехмерной области с вырезанным тонким телом // Докл. АН СССР. 1981. T. 256. № 1. С. 37-39.

14. Мазья В.Г., Назаров С.А., Пламеневский Б. А. Асимптотика решений задачи Дирихле в области с вырезанной тонкой трубкой // Матем. сб. 1981. Т. 116. № 2. С. 187-217.

15. Назаров C. A. Осреднение краевых задач в области, содержащей тонкую полость с периодически изменяющимся сечением // Труды ММО. 1990. Т. 53. С. 98-129.

16. Назаров C. A. Выводвариационного неравенства для формы малого приращения трещины отрьва // Механика твердого тела. 1989. № 2. С. 152-160.

17. Назаров C. A., Полякова O.P. Разрушение узкой перемычки между трещинами, лежащими в одной плоскости // Прикладная матем. и мех. 1991. Т. 55. № 1. С. 165-173.

18. Назаров C. А., Полякова О.Р. Об эквивалентности критериев разрушения для трещины отрыва в упругом пространстве // Механика твердого тела. 1992. № 2. С. 101-113.

19. Ван-Дайк М. Д. Методы возмущений в механике жидкости. М.: Мир, 1967.

20. Leguillon D., Sanches-Palencia E. Computation of singular solutions in elliptic problems and elasticity. Paris: Masson, 1987.

21. Ильин A. M. Согласование асимптотических разложений решений краевых задач. М.: Наука, 1989.

22. Nazarov S.A., Plamenevsky B.A. Elliptic problems in domains with piecewise smooth boundaries. Berlin: Walter de Gruyter, 1994.

23. Назаров C. A., Паукшто M. В. Дискретные модели и осреднения в теории упругости. Л.: Изд-во ЛГУ, 1984.

24. Mazja W. G., Nazarov S.A., Plamenevski B.A. Asymptotische Theorie elliptischer Randwertaufgaben in singular gestorten Gebieten. V. 1, 2. Berlin: Akadeemie-Verlag, $1990,1991$.

25. Назаров С.А., Пламеневский Б.А. Эллиптические задачи в областях с кусочно-гладкой границей. М.: Наука, 1991.

26. Ландкоф Н. С. Основы современной теории потенциала. М.: Наука, 1966.

27. Начель Ю. Об эквивалентных нормировках в функциональных пространствах // Вестник ЛГУ. 1974. № 7. С. 41-47.

28. Хёрмандер Л. Линейные дифференциальные операторы с частными производньми. М.: Мир, 1965.

29. Тейлор М. Псевдодифференциальные операторы. М.: Мир, 1985.

30. Мазья В. Г., Назаров С.А., Пламеневский Б. А. Об асимптотике решений эллиптических краевых задач при нерегулярном возмущении области // Проблемы матем. анализа. Вьп. 8. Л.: Изд-во ЛГУ, 1981. С. 72-153.

31. Agmon S., Douglis A., Nirenberg L. Estimates near the boundary for solutions of elliptic partial differential equations satisfying general boundary conditions. 1 // Comm. Pure Appl. Math. 1959. V. 12. № 4. P. 623-723.

32. Солонников B. A. Априорные оценки для уравнений второго порядка параболического типа // Труды МИАН. 1964. Т. 70. С. 133-212.

33. Levy H., Stampacchia G. On the regularity of the solution of a variational inequality // Comm. Pure Appl. Math. 1969. V. 22. P. 153-188.

34. Соболев С. Л. Уравнения математической физики. М.: Наука, 1966.

Санкт-Петербургский государственньй университет;

Государственная Морская Академия им. адмирала С. О. Макарова

Поступила в редакцию 10.02 .1995 